\title{
COMPUTING GREEN FUNCTIONS IN SMALL CHARACTERISTIC
}

\author{
MEINOLF GECK \\ To the memory of Kay Magaard
}

\begin{abstract}
Let $G(q)$ be a finite group of Lie type over a field with $q$ elements, where $q$ is a prime power. The Green functions of $G(q)$, as defined by Deligne and Lusztig, are known in almost all cases by work of Beynon-Spaltenstein, Lusztig und Shoji. Open cases exist for groups of exceptional type ${ }^{2} E_{6}, E_{7}, E_{8}$ in small characteristics. We propose a general method for dealing with these cases, which procedes by a reduction to the case where $q$ is a prime and then uses computer algebra techniques. In this way, all open cases in type ${ }^{2} E_{6}, E_{7}$ are solved, as well as at least one particular open case in type $E_{8}$.
\end{abstract}

\section{INTRODUCTION}

Let $G(q)$ be a group of Lie type over a finite field with $q$ elements. We are concerned with the problem of computing the Green functions of $G(q)$, as defined by Deligne and Lusztig [5]. This is an important and essential part of the more general problem of computing the whole character table of $G(q)$; see Carter's book [3] for further background. There is a long tradition of work on Green functions; the principal ideas and methods, which remain valid and state of the art as of today, are summarized in Shoji's survey [36] from 1987. At that point, the Green functions where known in all cases where $q$ is a power of a good prime for $G$, or where $q$ is arbitrary, $G$ is of small rank and the whole table of unipotent character values is available (like for $G_{2},{ }^{3} D_{4},{ }^{2} B_{2},{ }^{2} G_{2}$ ). Subsequently, explicit results for $F_{4}$, ${ }^{2} F_{4}, E_{6}$ in small characteristics were obtained by Malle [27], [28] and Porsch [33].

Regarding the general theory, it was first shown by Lusztig [22] (with some mild restrictions on $q$ ) and then by Shoji [37, [38] (in complete generality) that the original Green functions of 5 can be identified with another type of Green functions defined in terms of Lusztig's character sheaves [21]. This provides new, extremely powerful tools. In this setting, groups of classical type in characteristic 2 are dealt with by Shoji [4]. Thus, the remaining open cases are as follows:

$$
{ }^{2} E_{6}\left(3^{m}\right), \quad E_{7}\left(2^{m}\right), \quad E_{7}\left(3^{m}\right), \quad E_{8}\left(2^{m}\right), \quad E_{8}\left(3^{m}\right), \quad E_{8}\left(5^{m}\right)
$$

for any $m \geqslant 1$. (See Marcelo-Shinoda [29] for some comments about the Green functions of $F_{4}\left(3^{m}\right)$.) In principle, one could try to deal with these cases by similar methods as in the papers by Malle and Porsch mentioned above; however, these involve the technically complicated and unpleasant task of explicitly inducing class functions from proper subgroups. In this paper, we use another approach, similar to that in [11. By [12, Theorem 3.7], the computation of the Green functions of

Date: April 10, 2019.

1991 Mathematics Subject Classification. Primary 20C33; Secondary 20G40.

Key words and phrases. Finite groups of Lie type, Green functions, character sheaves. 
$G(q)$, where $q=p^{m}$ with $m \geqslant 1$, can be reduced to the base case where $m=1$ which amounts to just six individual cases which can be addressed by computer algebra methods. In this way, we will solve all the open cases for the groups of type ${ }^{2} E_{6}, E_{7}$ in the list ( $)$, as well as one particular case for type $E_{8}$ in characteristic 2.

In Section 2, we review the general plan for computing Green functions, which reduces matters to the determination of certain " $Y$-functions". In Section 3, we discuss a number of techniques for determining these functions. Consequently, we obtain a method for solving the remaining open problems which relies on knowing at least some values of the permutation character of $G(q)$ on the cosets of a Borel subgroup $B(q) \subseteq G(q)$. In order to compute such values, we shall work with an explicit realisation of $G(q)$ as a matrix group. In Section 4, we advertise a "canonical" way of constructing $G(q)$, following [8], 25]. Then the remaining sections deal with the discussion of the various cases in groups of type $F_{4}, E_{6},{ }^{2} E_{6}$, $E_{7}$; see Section 9 for the particular case in type $E_{8}$.

We heavily rely on Michel's version of CHEVIE [30], as well as programs (written by the author in GAP [6]) implementing the constructions in Section 4. As far as the remaining open cases in type $E_{8}$ are concerned, it seems that the above method might work in principle, but more sophisticated algorithms will certainly be required. (For example, one could replace the Borel subgroup $B(q)$ by a parabolic subgroup of $G(q)$.) This will be discussed elsewhere.

The main computational challenge of our approach is the explicit computation of the values of the above-mentioned permutation character of $G(q)$. For this purpose, we need to count the (left) cosets of $B(q)$ that are fixed by a given element $g \in G(q)$. But, because of the sheer size of the groups in question (e.g., for $G(q)=E_{7}(3)$ we have $[G(q): B(q)] \approx 17 \cdot 10^{30}$ ), it is entirely impossible to run through the complete list of cosets. Now a crucial feature of our approach is that, typically, we only need to obtain lower bounds for the number of fixed points, and this can be exploited as follows. By the sharp form of the Bruhat decomposition, we have a partition

$$
G(q)=\coprod_{w \in W} B(q) w B(q),
$$

where $W$ is the Weyl group of $G(q)$ and each double coset $B(q) w B(q)$ contains precisely $q^{l(w)}$ left $B(q)$-cosets; here, $l(w)$ is the length of $w$. Now we simply begin with various elements $w \in W$ of relatively small length, run through the left cosets that are contained in $B(q) w B(q)$, and check if they are fixed by $g$ or not. In a sense, we were just lucky because in all cases that we consider, this is sufficient to reach the desired lower bounds for the total number of fixed points - and there are cases where we never reached the exact total number, even after weeks or months of computations. (We will indicate the maximum length required for Weyl group elements in all cases in Sections 50.)

Acknowledgements. The author is indebted to Gunter Malle for a careful reading of the manuscript and for a number of useful comments. This work is a contribution to the SFB-TRR 195 "Symbolic Tools in Mathematics and their Application" of the German Research Foundation (DFG).

\section{On the Computation of Green functions}

Let $p$ be a prime and $k=\overline{\mathbb{F}}_{p}$ be an algebraic closure of the field with $p$ elements. Let $G$ be a connected reductive algebraic group over $k$ and assume that $G$ is defined 
over the finite subfield $\mathbb{F}_{q} \subseteq k$, where $q=p^{m}$ for some $m \geqslant 1$. Let $F: G \rightarrow G$ be the corresponding Frobenius map. Let $B_{0} \subseteq G$ be an $F$-stable Borel subgroup and $T_{0} \subseteq B_{0}$ be an $F$-stable maximal torus. Let $W=N_{G}\left(T_{0}\right) / T_{0}$ be the corresponding Weyl group. For each $w \in W$, let $R_{w}$ be the virtual representation of the finite group $G^{F}$ defined by Deligne-Lusztig [5, §1]. (In the setting of [3, $\S 7.2$ ], we have $\operatorname{Tr}\left(g, R_{w}\right)=R_{T_{w}, 1}(g)$ for $g \in G^{F}$, where $T_{w} \subseteq G$ is an $F$-stable maximal torus obtained from $T_{0}$ by twisting with $w$, and 1 stands for the trivial character of $T^{F}$.) This construction is carried out over $\overline{\mathbb{Q}}_{\ell}$, an algebraic closure of the $\ell$-adic numbers where $\ell$ is a prime not equal to $p$. The corresponding Green function is defined by

$$
Q_{w}: G_{\text {uni }}^{F} \rightarrow \overline{\mathbb{Q}}_{\ell}, \quad u \mapsto \operatorname{Tr}\left(u, R_{w}\right),
$$

where $G_{\text {uni }}$ denotes the set of unipotent elements of $G$. It is known that $Q_{w}(u) \in \mathbb{Z}$ for all $u \in G_{\text {uni }}^{F}$; see [3, $\left.\S 7.6\right]$. So the character formula [3, 7.2.8] shows that we also have $\operatorname{Tr}\left(g, R_{w}\right) \in \mathbb{Z}$ for all $g \in G^{F}$. The general plan for computing the values of $Q_{w}$ is explained in [20, Chap. 24], [36, §5], [39, 1.1-1.3] (even for generalised Green functions, which we will not consider here). In order to be able to address the main open issues, we will have to go through some of the steps of that plan, where we streamline the exposition as much as possible and concentrate on the algorithmic aspects.

Remark 2.1. The Frobenius map $F$ induces an automorphism of $W$ which we denote by $\gamma: W \rightarrow W$. Let $\operatorname{Irr}(W)$ be the set of irreducible representations of $W$ over $\overline{\mathbb{Q}}_{\ell}$ (up to isomorphism). Let $\operatorname{Irr}(W)^{\gamma}$ be the set of all those $E \in \operatorname{Irr}(W)$ that are $\gamma$-invariant, that is, there exists a bijective linear map $\sigma_{E}: E \rightarrow E$ such that $\sigma_{E} \circ w=\gamma(w) \circ \sigma_{E}: E \rightarrow E$ for all $w \in W$. Note that $\sigma_{E}$ is only unique up to scalar multiples but, if $\gamma$ has order $d \geqslant 1$, then one can always find $\sigma_{E}$ such that

$$
\sigma_{E}^{d}=\operatorname{id}_{E} \quad \text { and } \quad \operatorname{Tr}\left(\sigma_{E} \circ w, E\right) \in \mathbb{Z} \quad \text { for all } w \in W
$$

see [17, 3.2]. In what follows, we assume that a fixed choice of $\sigma_{E}$ satisfying the above conditions has been made for each $E \in \operatorname{Irr}(W)^{\gamma}$. (For example, one could take the "preferred" choice for $\sigma_{E}$ specified by Lusztig [19, 17.2].)

For $E \in \operatorname{Irr}(W)^{\gamma}$, the corresponding almost character is the class function $R_{E}: G^{F} \rightarrow \overline{\mathbb{Q}}_{\ell}$ defined by

$$
R_{E}(g):=\frac{1}{|W|} \sum_{w \in W} \operatorname{Tr}\left(\sigma_{E} \circ w, E\right) \operatorname{Tr}\left(g, R_{w}\right) \quad \text { for all } g \in G^{F} .
$$

Since all the terms $\operatorname{Tr}\left(\sigma_{E} \circ w, E\right)$ are integers, all the values $R_{E}$ are in $\mathbb{Q}$. By [17, 3.9], the above functions are orthonormal with respect to the standard inner product on class functions of $G^{F}$. Furthermore, by [16, 3.19], we have

$$
Q_{w}(u)=\sum_{E \in \operatorname{Irr}(W)^{\gamma}} \operatorname{Tr}\left(\sigma_{E} \circ w, E\right) R_{E}(u) \quad \text { for } w \in W, u \in G_{\mathrm{uni}}^{F}
$$

Hence, knowing the values of all Green functions $Q_{w}$ is equivalent to knowing the values of all $R_{E}$ on $G_{\text {uni }}^{F}$. We define the matrix $\tilde{\Omega}=\left(\tilde{\omega}_{E^{\prime}, E}\right)_{E^{\prime}, E \in \operatorname{Irr}(W)^{\gamma}}$ where

$$
\tilde{\omega}_{E^{\prime}, E}:=\frac{1}{|W|} \sum_{w \in W}\left[G^{F}: T_{w}^{F}\right] \operatorname{Tr}\left(\sigma_{E^{\prime}} \circ w, E^{\prime}\right) \operatorname{Tr}\left(\sigma_{E} \circ w, E\right) \in \mathbb{Q} ;
$$

here, $T_{w} \subseteq G$ denotes an $F$-stable maximal torus obtained from $T_{0}$ by twisting with $w$ and the maps $\sigma_{E}: E \rightarrow E, \sigma_{E^{\prime}}: E^{\prime} \rightarrow E^{\prime}$ are as above. 
Proposition 2.2 (Orthogonality relations). For $E, E^{\prime} \in \operatorname{Irr}(W)^{\gamma}$, we have

$$
\tilde{\omega}_{E^{\prime}, E}=\sum_{g \in G_{\mathrm{uni}}^{F}} R_{E^{\prime}}(g) R_{E}(g) .
$$

Proof. Arguing as in [16, 3.19], the above relations are a formal consequence of the orthogonality relations for the Green functions $Q_{w}$ in [3, Prop. 7.6.2].

Let $\mathscr{N}_{G}$ be the set of all pairs $(C, \mathscr{E})$ where $C$ is a unipotent class in $G$ and $\mathscr{E}$ is a $G$-equivariant irreducible $\overline{\mathbb{Q}}_{\ell}$-local system on $C$ (up to isomorphism). The Springer correspondence defines an injective map

$$
\iota_{G}: \operatorname{Irr}(W) \hookrightarrow \mathscr{N}_{G} ;
$$

see Lusztig [18], [20, Chap 24], and the references there. Let $E \in \operatorname{Irr}(W)$ and $\iota_{G}(E)=(C, \mathscr{E}) \in \mathscr{N}_{G}$. Then we define

$$
d_{E}:=\left(\operatorname{dim} G-\operatorname{dim} C-\operatorname{dim} T_{0}\right) / 2 .
$$

Note that $\operatorname{dim} C_{G}(g) \geqslant \operatorname{dim} T_{0}$ for $g \in G$. Furthermore, $d_{E} \in \mathbb{Z}_{\geqslant 0}$ since $\operatorname{dim} G-$ $\operatorname{dim} T_{0}$ is always even and so is $\operatorname{dim} C$; see [3, §5.10] and the references there. Now assume that $E \in \operatorname{Irr}(W)^{\gamma}$. Then $F(C)=C$ and $F^{*} \mathscr{E} \cong \mathscr{E}$. We define a function

$$
Y_{E}: G_{\text {uni }}^{F} \rightarrow \mathbb{Q}
$$

as follows. Let $g \in G_{\text {uni }}^{F}$. Then we set $Y_{E}(g):=0$ if $g \notin C$, and

$$
Y_{E}(g):=q^{-d_{E}} R_{E}(g) \quad \text { if } g \in C^{F} \text {. }
$$

Now we can state the following fundamental result.

Theorem 2.3 (Lusztig, Shoji). In the above setting, the following hold.

(a) The functions $\left\{Y_{E} \mid E \in \operatorname{Irr}\left(W^{\gamma}\right)\right\}$ are integer-valued and linearly independent.

(b) There are unique coefficients $p_{E^{\prime}, E} \in \mathbb{Z}\left(E^{\prime}, E \in \operatorname{Irr}(W)^{\gamma}\right)$ such that

$$
\left.R_{E}\right|_{G_{\mathrm{uni}}^{F}}=\sum_{E^{\prime} \in \operatorname{Irr}(W)^{\gamma}} q^{d_{E}} p_{E^{\prime}, E} Y_{E^{\prime}} \quad \text { for all } E \in \operatorname{Irr}(W)^{\gamma} .
$$

(c) We have $p_{E, E}=1$; furthermore, $p_{E^{\prime}, E}=0$ if $E^{\prime} \neq E$ and $d_{E^{\prime}} \geqslant d_{E}$.

Proof. By Lusztig [22] (with some mild restrictions on $p, q$ ) and Shoji [37], 38] (in complete generality), the original Green functions of [5] can be identified with another type of Green functions defined in terms of character sheaves [21]. So we can place ourselves in the setting of [20, Chap. 24]. Thus, the restrictions of the functions $R_{E}$ to $G_{\text {uni }}^{F}$ are indeed the characteristic functions of the character sheaves $A_{i}$ in [20, 24.2]. Furthermore, the functions $Y_{E}$ defined above are indeed equal to the functions $Y_{i}$ in $[20,24.2 .3]$; thus, if $\iota_{G}(E)=(C, \mathscr{E})$, then we have

$$
Y_{E}(g)=\operatorname{Tr}\left(\psi_{g}, \mathscr{E}_{g}\right) \quad \text { for } g \in C^{F},
$$

where $\mathscr{E}_{g}$ is the stalk of $\mathscr{E}$ at $g$ and $\psi_{g}: \mathscr{E}_{g} \rightarrow \mathscr{E}_{g}$ is a certain linear map of finite order. In particular, this shows that the values of $Y_{E}$ are algebraic integers. Then all of the above statements follow from [20, 24.2, 24.3, 24.5] and [20, Theorem 24.4]. Note that the hypotheses of [20, Theorem 24.4] ("cleanness") are always satisfied by the main result of [24]. (Since we are only dealing with Green functions of $G^{F}$, and not with generalised Green functions, it would actually be sufficient to refer 
to [7, §3] instead of [24]; see also [12, §2] where all of the above are discussed in somewhat more detail.)

Remark 2.4. Lusztig [20, §24.4] describes a purely combinatorial algorithm for computing the coefficients $p_{E^{\prime}, E}$, which modifies and simplifies an earlier algorithm of Shoji [36, §5]. For this purpose, we define three matrices

$$
P=\left(p_{E^{\prime}, E}\right), \quad \Omega=\left(\omega_{E^{\prime}, E}\right), \quad \Lambda=\left(\lambda_{E^{\prime}, E}\right),
$$

where, in each case, the indices run over all $E^{\prime}, E \in \operatorname{Irr}(W)^{\gamma}$. Here, $p_{E^{\prime}, E}$ are the coefficients in Theorem 2.3, furthermore,

$$
\omega_{E^{\prime}, E}:=q^{-d_{E}-d_{E^{\prime}}} \tilde{\omega}_{E^{\prime}, E} \quad \text { and } \quad \lambda_{E^{\prime}, E}:=\sum_{g \in G_{\mathrm{uni}}^{F}} Y_{E^{\prime}}(g) Y_{E}(g) .
$$

Then the orthogonality relations in Proposition 2.2 give rise to the matrix identity

$$
P^{\operatorname{tr}} \cdot \Lambda \cdot P=\Omega ; \quad \text { see Lusztig [20, 24.9], Shoji [36, 5.6]. }
$$

In general, given the right hand side $\Omega$, such a system of equations will not have a unique solution for $P, \Lambda$. But if we take into account the additional information on the coefficients $p_{E^{\prime}, E}$ in Theorem 2.3(c), then it does have a unique solution, which can be found by a recursive algorithm.

Remark 2.5. The Springer correspondence is explicitly known in all cases; see the tables in Carter [3, §13.3], Lusztig [18], Lusztig-Spaltenstein [26], Spaltenstein [41] (and the further references there). It can be obtained electronically, via tables or combinatoral algorithms, through Michel's version of the CHEVIE system [30]; see the function UnipotentClasses. There is also an implementation of the algorithm in Remark 2.4, see the function ICCTable. Examples will be given below.

Example 2.6. Let $E_{1} \in \operatorname{Irr}(W)$ be the trivial representation of $W$. Clearly, we have $E_{1} \in \operatorname{Irr}(W)^{\gamma}$; furthermore, we can certainly choose $\sigma_{E_{1}}: E_{1} \rightarrow E_{1}$ to be the identity map. Then, with this choice, we have

$$
R_{E_{1}}(g)=1 \quad \text { for all } g \in G^{F}
$$

see [5, 7.14.1] or [3, Prop 7.4.2]. It is also known that $\iota_{G}\left(E_{1}\right)=\left(C_{\text {reg}}, \overline{\mathbb{Q}}_{\ell}\right)$ where $C_{\text {reg }}$ is the class of regular unipotent elements (see, e.g., [41, 1.1]); hence, we have

$$
d_{E_{1}}=0 \quad \text { and }\left.\quad R_{E_{1}}\right|_{G_{\text {uni }}^{F}}=\sum_{E^{\prime} \in \operatorname{Irr}(W)^{\gamma}} p_{E^{\prime}, E} Y_{E^{\prime}}
$$

Combining the above two expressions for $R_{E_{1}}$, the functions $Y_{E^{\prime}}$ are determined for all $E^{\prime} \in \operatorname{Irr}(W)^{\gamma}$ such that $p_{E^{\prime}, E_{1}} \neq 0$. Indeed, if $p_{E^{\prime}, E_{1}} \neq 0$, then let us write $\iota_{G}\left(E^{\prime}\right)=\left(C^{\prime}, \mathscr{E}^{\prime}\right)$ where $C^{\prime}$ is an $F$-stable unipotent class. Since the functions $\left\{Y_{E} \mid E \in \operatorname{Irr}\left(W^{\gamma}\right)\right\}$ are linearly independent, we conclude that

$$
p_{E^{\prime}, E_{1}} Y_{E^{\prime}}(g)=1 \quad \text { for all } g \in C^{\prime F} \text {. }
$$

Since $p_{E^{\prime}, E} \in \mathbb{Z}$ and $Y_{E^{\prime}}(g) \in \mathbb{Z}$ for all $g \in G^{F}$, we either have $Y_{E^{\prime}}(g)=1$ for all $g \in C^{\prime F}$, or $Y_{E^{\prime}}(g)=-1$ for all $g \in C^{\prime F}$, where the sign is determined by $p_{E^{\prime}, E_{1}}$.

Example 2.7. Let $G$ be of type $G_{2}$ and $p=3$. In this case, $W=\left\langle s_{1}, s_{2}\right\rangle$ where $s_{1}$ is the reflection corresponding to a long simple root and $s_{1}$ is the reflection corresponding to a short simple root. We have

$$
\operatorname{Irr}(W)=\left\{E_{1,0}, E_{1,6}, E_{1,3}^{\prime}, E_{1,3}^{\prime \prime}, E_{2,1}, E_{2,2}\right\}
$$


TABLE 1. The Springer correspondence and $p_{E^{\prime}, E}$ for $G_{2}, p=3$

\begin{tabular}{cccc}
\hline$E$ & $d_{E}$ & $A(u)$ & $\iota_{G}(E)$ \\
\hline$E_{1,6}$ & 6 & $\{1\}$ & $\left(1, \overline{\mathbb{Q}}_{\ell}\right)$ \\
$E_{1,3}^{\prime}$ & 3 & $\{1\}$ & $\left(\left(\tilde{A}_{1}\right)_{3}, \overline{\mathbb{Q}}_{\ell}\right)$ \\
$E_{1,3}^{\prime \prime}$ & 3 & $\{1\}$ & $\left(A_{1}, \overline{\mathbb{Q}}_{\ell}\right)$ \\
$E_{2,2}$ & 2 & $\{1\}$ & $\left(\tilde{A}_{1}, \overline{\mathbb{Q}}_{\ell}\right)$ \\
$E_{2,1}$ & 1 & $\mathbb{Z} / 2 \mathbb{Z}$ & $\left(G_{2}\left(a_{1}\right), \overline{\mathbb{Q}}_{\ell}\right)$ \\
$E_{1,0}$ & 0 & $\mathbb{Z} / 3 \mathbb{Z}$ & $\left(G_{2}, \overline{\mathbb{Q}}_{\ell}\right)$ \\
\hline
\end{tabular}

\begin{tabular}{ccccccc}
\hline$p_{E^{\prime}, E}$ & $E_{1,6}$ & $E_{1,3}^{\prime}$ & $E_{1,3}^{\prime \prime}$ & $E_{2,2}$ & $E_{2,1}$ & $E_{1,0}$ \\
\hline$E_{1,6}$ & 1 & 1 & 1 & $q^{2}+1$ & $q^{4}+1$ & 1 \\
$E_{1,3}^{\prime}$ & 0 & 1 & 0 & 1 & 1 & 1 \\
$E_{1,3}^{\prime \prime}$ & 0 & 0 & 1 & 1 & 1 & 1 \\
$E_{2,2}$ & 0 & 0 & 0 & 1 & 1 & 1 \\
$E_{2,1}$ & 0 & 0 & 0 & 0 & 1 & 1 \\
$E_{1,0}$ & 0 & 0 & 0 & 0 & 0 & 1 \\
\hline
\end{tabular}

where $E_{1,0}$ is the trivial representation, $E_{1,6}$ is the $\operatorname{sign}$ representations, $E_{1,3}^{\prime}, E_{1,3}^{\prime \prime}$ are two further one-dimensional representations such that

$$
\operatorname{Tr}\left(s_{1}, E_{1,3}^{\prime}\right)=\operatorname{Tr}\left(s_{2}, E_{1,3}^{\prime \prime}\right)=-1 \quad \text { and } \quad \operatorname{Tr}\left(s_{1}, E_{1,3}^{\prime \prime}\right)=\operatorname{Tr}\left(s_{2}, E_{1,3}^{\prime}\right)=1 ;
$$

finally, $E_{2,1}$ is the standard reflection representation and $E_{2,2}$ is a further twodimensional representation. The Frobenius map $F$ acts trivially on $W$ and so $\gamma=$ $\operatorname{id}_{W}$. In Michel's version of CHEVIE [30], we obtain the information on unipotent classes and the Springer correspondence as follows.

$$
\begin{aligned}
& \text { gap> } W:=\text { CoxeterGroup ("G" }, 2) ; \\
& \text { gap> uc:= UnipotentClasses }(W, 3) ; ; \quad \text { p }=3 \\
& \text { gap> Display(uc); Display (ICCTable (uc)); }
\end{aligned}
$$

The information is summarized in Table 1 (see also [41, p. 329]). There are 6 unipotent classes, denoted by $G_{2}, G_{2}\left(a_{1}\right), \tilde{A}_{1}, A_{1},\left(\tilde{A}_{1}\right)_{3}, 1$. Since $\gamma=\mathrm{id} W$, we also have $\sigma_{E}=\operatorname{id}_{E}$ for all $E \in \operatorname{Irr}(W)$. Thus, we obtain explicit expressions

$$
\left.R_{E}\right|_{G_{\mathrm{uni}}^{F}}=\sum_{E^{\prime} \in \operatorname{Irr}(W)} q^{d_{E}} p_{E^{\prime}, E} Y_{E^{\prime}} \quad \text { for all } E \in \operatorname{Irr}(W) .
$$

It remains to determine the values of $Y_{E}$ for all $E \in \operatorname{Irr}(W)$. In the present case, this is easily done using Example 2.6. Indeed, since all entries in the last column of Table 1 are equal to 1, we have $R_{E_{1,0}}=\sum_{E \in \operatorname{Irr}(W)} Y_{E}$. Hence, each function $Y_{E}$ is identically 1 on $C^{F}$ where $\iota_{G}(E)=\left(C, \overline{\mathbb{Q}}_{\ell}\right)$.

In general, the determination of the functions $Y_{E}$ is a very subtle problem. In order to solve it, one either needs further geometric information (as, for example, in Beynon-Spaltenstein [1, §3, Case V], Shoji [40, §1]) or some additional information about character values of $G^{F}$, which was readily availaible in the above example but may require much more work in other cases (as, for example, in Malle [28]). The following discussion, which is inspired by the approach of Marcelo-Shinoda [29], will turn out to be very useful in later sections.

Remark 2.8. For $w=1$, the virtual representation $R_{1}$ of Deligne-Lusztig is known to be an actual representation, which is in fact the permutation representation of $G^{F}$ on the cosets of $B_{0}^{F}$ (see [5, 1.5] or [3, 7.2.4]). Thus, for any unipotent element $u \in G^{F}$, we have

$$
\begin{aligned}
Q_{1}(u) & =\operatorname{Tr}\left(u, R_{1}\right)=\left|\left\{g B_{0}^{F} \in G^{F} / B_{0}^{F} \mid u g B_{0}^{F}=g B_{0}^{F}\right\}\right| \\
& =\left|\left\{g B_{0}^{F} \in G^{F} / B_{0}^{F} \mid g^{-1} u g \in B_{0}^{F}\right\}\right|=\sum_{1 \leqslant i \leqslant r} \frac{\left|C_{G}(u)^{F}\right|}{\left|C_{B_{0}}\left(u_{i}\right)^{F}\right|}
\end{aligned}
$$


where $u_{1}, \ldots, u_{r} \in B_{0}^{F}$ are representatives of the conjugacy classes of $B_{0}^{F}$ that are contained in the $G^{F}$-conjugacy class of $u$. On the other hand, expressing $R_{1}$ as a linear combination of $R_{E}$ 's and then using Theorem 2.3 (b), we obtain that

$$
Q_{1}(u)=\sum_{E^{\prime} \in \operatorname{Irr}(W)^{\gamma}} \tilde{p}_{E^{\prime}} Y_{E^{\prime}}(u) \quad \text { where } \quad \tilde{p}_{E^{\prime}}:=\sum_{E \in \operatorname{Irr}(W)^{\gamma}} q^{d_{E}} \operatorname{Tr}\left(\sigma_{E}\right) p_{E^{\prime}, E} .
$$

Thus, since the terms $\tilde{p}_{E^{\prime}}$ are determined by the algorithm in Remark 2.4, this yields conditions on the values of the functions $Y_{E^{\prime}}$, once we manage to obtain some information about $Q_{1}(u)$ by other means.

In Sections 5] 8, we will try to evaluate $Q_{1}(u)$ explicitly for certain unipotent elements $u$ (see Example 3.6 below for a first illustration). For this purpose, we note that the formula for $Q_{1}(u)$ can be further refined using the Bruhat decomposition. Let $\Phi$ be the root system of $G$ with respect to $T_{0}$. Let $\Phi^{+} \subseteq \Phi$ be the set of positive roots determined by the choice of $B_{0}$. Finally, let $\left\{\alpha_{i} \mid i \in I\right\} \subseteq \Phi^{+}$be the corresponding set of simple roots, where $I$ is a finite indexing set. This defines a length function $l: W \rightarrow \mathbb{Z}_{\geqslant 0}$. For each $\alpha \in \Phi$, let $U_{\alpha}=\left\{x_{\alpha}(t) \mid t \in k\right\} \subseteq G$ be the corresponding root subgroup. For $w \in W$, we set

$$
U_{w}:=\left\langle U_{\alpha} \mid \alpha \in \Phi_{w}^{+}\right\rangle \subseteq G \quad \text { where } \quad \Phi_{w}^{+}:=\left\{\alpha \in \Phi^{+} \mid w(\alpha) \in \Phi^{-}\right\} ;
$$

we also fix a representative $\dot{w}$ of $w$ in $N_{G}\left(T_{0}\right)$. Here, we tacitly assume that $\dot{w}$ is chosen such that $F(\dot{w})=\dot{w}$ whenever $\gamma(w)=w$, which is possible by [3, p. 33]. Then we have the following sharp form of the Bruhat decomposition.

$$
G=\coprod_{w \in W} U_{w} \dot{w} B_{0} \quad \text { (with uniqueness of expression); }
$$

see [3. Theorem 2.5.14 and Prop. 2.5.6]. Writing $\Phi_{w}^{+}=\left\{\beta_{1}, \ldots, \beta_{l}\right\}$ where $l=l(w)$, we actually have $U_{w}=U_{\beta_{1}} \cdots U_{\beta_{l}}$ with uniqueness of expression.

Lemma 2.9. Let $u \in G^{F}$ be unipotent. Then

$$
Q_{1}(u)=\sum_{w \in W, \gamma(w)=w}\left|Q_{1, w}(u)\right|
$$

where $Q_{1, w}(u):=\left\{v \in U_{w}^{F} \mid \dot{w}^{-1} v^{-1} u v \dot{w} \in B_{0}^{F}\right\}$ for all $w \in W$ such that $\gamma(w)=w$.

Proof. By [3, §2.9], we also have a sharp form of the Bruhat decomposition for the finite group $G^{F}$, such that $G^{F}=\coprod_{w} U_{w}^{F} \dot{w} B_{0}^{F}$, where the union runs over all $w \in W$ such that $\gamma(w)=w$. Inverting elements, we see that

$$
\left\{v \dot{w}^{-1} \mid w \in W, \gamma(w)=w, v \in U_{w}^{F}\right\}
$$

is a complete set of representatives of the cosets $\left\{g B_{0}^{F} \mid g \in G^{F}\right\}$. This yields the above formula.

Remark 2.10. As far as explicit computations using a computer are concerned, the above formula means that

$$
Q_{1}(u) \geqslant \sum_{w}\left|Q_{1, w}(u)\right|
$$

where $w$ runs over all elements of $W$ of any given bounded length. Note that $\left|U_{w}^{F}\right|=q^{l(w)}$ (see [3, p. 74]) which quickly becomes very large with growing $l(w)$. Thus, we can only reasonably work with bounds like $l(w) \leqslant 25$ (if $q=2$ ) or 
$l(w) \leqslant 16$ (if $q=3$ ) on a standard computer. In any case, the above estimate will be crucial in our discussion of groups of exceptional type in Sections 5 , 9 .

\section{ON THE DETERMinAtion OF THE FUnCTIONS $Y_{E}$}

We will assume from now on that $G$ is simple and that the Frobenius map $F: G \rightarrow G$ is given by

$$
F=\tilde{\gamma} \circ F_{p}^{m}=F_{p}^{m} \circ \tilde{\gamma} \quad(m \geqslant 1)
$$

where $\tilde{\gamma}: G \rightarrow G$ is an automorphism of finite order (leaving $T_{0}, B_{0}$ invariant) and $F_{p}: G \rightarrow G$ is a Frobenius map corresponding to a split $\mathbb{F}_{p}$-rational structure, such that $F_{p}$ commutes with $\tilde{\gamma}$ and $F_{p}(t)=t^{p}$ for all $t \in T_{0}$. Thus, $G^{F}$ is an untwisted or twisted Chevalley group, as in Steinberg [42]. Note that $\tilde{\gamma}$ induces an automorphism of $W$ which is just the automorphism $\gamma: W \rightarrow W$ induced by $F$ considered earlier.

Remark 3.1. It is known that all unipotent classes of $G$ are $F_{p}$-stable (since, in each case, representatives of the classes are known which lie in $G^{F_{p}}=G\left(\mathbb{F}_{p}\right)$; see, e.g., Liebeck-Seitz [15]). Let $C$ be an $F$-stable unipotent class. We shall also make the following assumption.

(a) There exists an element $u_{0} \in C^{F}$ such that $F$ acts trivially on the finite group of components $A\left(u_{0}\right):=C_{G}\left(u_{0}\right) / C_{G}^{\circ}\left(u_{0}\right)$.

If $(\mathbf{\beta})$ holds, then there is a bijective correspondence between the conjugacy classes of $A\left(u_{0}\right)$ and the conjugacy classes of $G^{F}$ that are contained in the set $C^{F}$ (see, e.g., [15. Lemma 2.12]). For $a \in A\left(u_{0}\right)$, an element in the corresponding $G^{F}$-conjugacy class is given by $u_{a}=h u_{0} h^{-1}$ where $h \in G$ is such that $h^{-1} F(h) \in C_{G}\left(u_{0}\right)$ maps to $a$ under the natural homomorphism $C_{G}\left(u_{0}\right) \rightarrow A\left(u_{0}\right)$. (The existence of $h$ is guaranteed by Lang's Theorem; note that $h$ is not unique but $u_{a}=h u_{0} h^{-1}$ is well-defined up to $G^{F}$-conjugacy.)

Remark 3.2. Let $E \in \operatorname{Irr}(W)^{\gamma}$ and $\iota_{G}(E)=(C, \mathscr{E}) \in \mathscr{N}_{G}$, such that $F(C)=C$ and $F^{*} \mathscr{E} \cong \mathscr{E}$. Now let us fix an element $u_{0} \in C^{F}$ as in (⿻), such that $F$ acts trivially on $A\left(u_{0}\right)$. Then it is known (see Lusztig [23, 19.7]) that there is a natural $A\left(u_{0}\right)$-module structure on the stalk $\mathscr{E}_{u_{0}}$; in fact, we have $\mathscr{E}_{u_{0}} \in \operatorname{Irr}\left(A\left(u_{0}\right)\right)$ and there is a root of unity $\delta_{E} \in \overline{\mathbb{Q}}_{\ell}$ such that

$$
Y_{E}\left(u_{a}\right)=\delta_{E} \operatorname{Tr}\left(a, \mathscr{E}_{u_{0}}\right) \quad \text { for all } a \in A\left(u_{0}\right) .
$$

Since the values of $Y_{E}$ are integers, it easily follows that $\delta_{E}= \pm 1$. (See [12, Lemma 3.3] for further details.) Note that $\operatorname{Tr}\left(a, \mathscr{E}_{u_{0}}\right)$ is just an entry in the ordinary character table of $A\left(u_{0}\right)$. In particular, if $a=1$, then $u_{1}$ is $G^{F}$-conjugate to $u_{0}$ and so $\delta_{E}$ is determined by the identity

$$
Y_{E}\left(u_{0}\right)=\delta_{E} \operatorname{dim} \mathscr{E}_{u_{0}}
$$

Thus, the whole problem of computing the Green functions $Q_{w}$ is reduced to the determination of the signs $\delta_{E}= \pm 1$ for $E \in \operatorname{Irr}(W)^{\gamma}$ (cf. Shoji [39, 1.3, p. 161]).

Remark 3.3. In the tables in Carter [3, §13.3], Lusztig-Spaltenstein [26] and Spaltenstein [41], the pair $(C, \mathscr{E}) \in \mathscr{N}_{G}$ corresponding to $E \in \operatorname{Irr}(W)$ via the Springer correspondence is specified by indicating the class $C$, the group $A(u)$ (where $u \in C$ ) and the irreducible $A(u)$-module $\mathscr{E}_{u}$. For example, in Table 1, we have $\mathscr{E} \cong \overline{\mathbb{Q}}_{\ell}$ and so $\mathscr{E}_{u}$ is the trivial representation of $A(u)$, in all cases. For 
$G$ of exceptional type, the possibilities for $A(u)$ are rather limited: either $A(u)$ is abelian of order at most 6 , or a dihedral group of order 8 , or isomorphic to a symmetric group $\mathfrak{S}_{r}$ where $r=3,4,5$, or isomorphic to $\mathbb{Z} / 2 \mathbb{Z} \times \mathfrak{S}_{3}$ (see [41, 5.4]).

Remark 3.4. Let $C$ be an $F$-stable unipotent class and $u_{0} \in C^{F}$ be such that $F$ acts trivially on $A\left(u_{0}\right)$. Let $u_{0}=u_{1}, u_{2}, \ldots, u_{r} \in C^{F}$ be representatives of the $G^{F}$-conjugacy classes that are contained in $C^{F}$, and let $a_{1}, \ldots, a_{r} \in A\left(u_{0}\right)$ be corresponding representatives of the conjugacy classes of $A\left(u_{0}\right)$ (see Remark 3.1).

(a) Let $E_{0} \in \operatorname{Irr}(W)^{\gamma}$ be such that $\iota_{G}\left(E_{0}\right)=\left(C, \overline{\mathbb{Q}}_{\ell}\right)$. Then the corresponding $A\left(u_{0}\right)$-module is the trivial representation and we have

$$
Y_{E_{0}}\left(u_{i}\right)=\delta_{E_{0}} \quad \text { for } 1 \leqslant i \leqslant r .
$$

Now let $E_{1} \in \operatorname{Irr}(W)$ be the trivial representation. Then the restriction of the almost character $R_{E_{1}}$ to $C^{F}$ is constant and so $p_{E_{0}, E_{1}} \delta_{E_{0}}=1$ (see Example 2.6). Hence, the sign $\delta_{E_{0}}$ is determined by the Lusztig-Shoji algorithm in Remark 2.4.

(b) Let $E \in \operatorname{Irr}(W)^{\gamma}$ be such that $\iota_{G}\left(E_{0}\right)=(C, \mathscr{E})$ where $\mathscr{E}$ is not the trivial local system. Then we have $Y_{E}\left(u_{i}\right)=\delta_{E} \operatorname{Tr}\left(a_{i}, \mathscr{E}_{u_{0}}\right)$ for $1 \leqslant i \leqslant r$. Hence, we obtain

$$
\lambda_{E_{0}, E}=\sum_{g \in G^{F}} Y_{E_{0}}(g) Y_{E}(g)=\delta_{E_{0}} \delta_{E} \sum_{1 \leqslant i \leqslant r}\left[G^{F}: C_{G}\left(u_{i}\right)^{F}\right] \operatorname{Tr}\left(a_{i}, \mathscr{E}_{u_{0}}\right) .
$$

The sum on the right hand can be explicitly computed using the knowledge of the centraliser orders $\left|C_{G}\left(u_{i}\right)^{F}\right|$ and the character table of the group $A\left(u_{0}\right)$. On the other hand, the left hand side is also known from the Lusztig-Shoji algorithm in Remark 2.4. Hence, if the left hand side is non-zero, then we also obtain $\delta_{E_{0}} \delta_{E}$; since $\delta_{E_{0}}$ is known from (a), this also determines $\delta_{E}$.

If the left hand side is zero, then some special arguments are required. A very particular such case occurs for $G$ of type $E_{8}$ and $p \neq 2,3$, where $A\left(u_{0}\right) \cong \mathfrak{S}_{3}$ and it turns out that $\delta_{E_{0}}=1$ and $\delta_{E} \equiv q \bmod 3$; see Beynon-Spaltenstein [1, §3, Case 5]. (We will encounter a similar case in Section 9.)

Example 3.5. Let $C$ be an $F$-stable unipotent class such that $A(u) \cong \mathbb{Z} / 2 \mathbb{Z}$ for $u \in C$. Note that $F$ acts trivially on $A(u)$ for any $u \in C^{F}$ (since $A(u)$ has order 2 ). So let us fix some $u_{0} \in C^{F}$. Assume that $E_{0} \in \operatorname{Irr}(W)^{\gamma}$ is such that $\iota_{G}\left(E_{0}\right)=$ $\left(C, \overline{\mathbb{Q}}_{\ell}\right)$. Then the corresponding sign $\delta_{E_{0}}$ is determined as in Remark 3.4(a). Let us also assume that there exists $E \in \operatorname{Irr}(W)^{\gamma}$ such that $\iota_{G}(E)=(C, \mathscr{E})$ where $\mathscr{E}$ is a non-trivial local system. Now $C^{F}$ splits into two classes in $G^{F}$; let $u_{0}^{\prime} \in C^{F}$ be such that $u_{0}, u_{0}^{\prime}$ are not conjugate in $G^{F}$. Then the values of $Y_{E_{0}}, Y_{E}$ are given by

$$
Y_{E_{0}}\left(u_{0}\right)=Y_{E_{0}}\left(u_{0}^{\prime}\right)=\delta_{E} \quad \text { and } \quad Y_{E}\left(u_{0}\right)=\delta_{E}, Y_{E}\left(u_{0}^{\prime}\right)=-\delta_{E} .
$$

(Note that $\overline{\mathbb{Q}}_{\ell}$ corresponds to the trivial character of $A\left(u_{0}\right)$ and $\mathscr{E}$ corresponds to the non-trivial character of $A\left(u_{0}\right)$.) Now, we either have $\delta_{E}=\delta_{E_{0}}$ or $\delta_{E}=-\delta_{E_{0}}$. But, as already discussed in [1, p. 591], if we are in the second case, then we change the roles of $u_{0}, u_{0}^{\prime}$ and, with the new choice of $u_{0}$, we will have $\delta_{E}=\delta_{E_{0}}$. Thus, in the present situation, we can always choose $u_{0} \in C^{F}$ such that $\delta_{E}=\delta_{E_{0}}$, and $u_{0}$ is unique up to conjugation within $G^{F}$. The only remaining problem is to identify $u_{0}$ in a given list of representatives of unipotent classes. In order to try to solve this problem, we follow Remark 3.4(b) and consider the relation

$$
\lambda_{E_{0}, E}=\sum_{g \in G^{F}} Y_{E_{0}}(g) Y_{E}(g)=\left|G^{F}\right|\left(\left|C_{G}\left(u_{0}\right)^{F}\right|^{-1}-\left|C_{G}\left(u_{0}^{\prime}\right)^{F}\right|^{-1}\right) \delta_{E_{0}} \delta_{E} .
$$


This leads to the following two cases.

(a) If $\lambda_{E_{0}, E} \neq 0$, then $(*)$ implies $\left|C_{G}\left(u_{0}\right)^{F}\right| \neq\left|C_{G}\left(u_{0}^{\prime}\right)^{F}\right|$, which distinguishes the representatives $u_{0}, u_{0}^{\prime}$.

(b) If $\lambda_{E_{0}, E}=0$, then an additional argument is required in order to distinguish the representatives $u_{0}, u_{0}^{\prime}$. (See $\$ 5.2$ below for a typical example.)

TABLE 2. The Springer correspondence and $p_{E^{\prime}, E}$ for $G_{2}, p \neq 3$

\begin{tabular}{cccc}
\hline$E$ & $d_{E}$ & $A(u)$ & $\iota_{G}(E)$ \\
\hline$E_{1,6}$ & 6 & $\{1\}$ & $\left(1, \overline{\mathbb{Q}}_{\ell}\right)$ \\
$E_{1,3}^{\prime \prime}$ & 3 & $\{1\}$ & $\left(A_{1}, \overline{\mathbb{Q}}_{\ell}\right)$ \\
$E_{2,2}$ & 2 & $\{1\}$ & $\left(\tilde{A}_{1}, \overline{\mathbb{Q}}_{\ell}\right)$ \\
$E_{2,1}$ & 1 & $\mathfrak{S}_{3}$ & $\left(G_{2}\left(a_{1}\right), \overline{\mathbb{Q}}_{\ell}\right)$ \\
$E_{1,3}^{\prime}$ & 1 & $\mathfrak{S}_{3}$ & $\left(G_{2}\left(a_{1}\right), \mathscr{E}\right)$ \\
$E_{1,0}$ & 0 & $\mathbb{Z} /(p, 2) \mathbb{Z}$ & $\left(G_{2}, \overline{\mathbb{Q}}_{\ell}\right)$ \\
\hline
\end{tabular}

\begin{tabular}{ccccccc}
\hline$p_{E^{\prime}, E}$ & $E_{1,6}$ & $E_{1,3}^{\prime \prime}$ & $E_{2,2}$ & $E_{2,1}$ & $E_{1,3}^{\prime}$ & $E_{1,0}$ \\
\hline$E_{1,6}$ & 1 & 1 & $q^{2}+1$ & $q^{4}+1$ & $q^{2}$ & 1 \\
$E_{1,3}^{\prime \prime}$ & 0 & 1 & 1 & 1 & 0 & 1 \\
$E_{2,2}$ & 0 & 0 & 1 & 1 & 1 & 1 \\
$E_{2,1}$ & 0 & 0 & 0 & 1 & 0 & 1 \\
$E_{1,3}^{\prime}$ & 0 & 0 & 0 & 0 & 1 & 0 \\
$E_{1,0}$ & 0 & 0 & 0 & 0 & 0 & 1
\end{tabular}

(where $\mathscr{E} \not \approx \overline{\mathbb{Q}}_{\ell}, \operatorname{dim} \mathscr{E}_{u}=2$ )

Example 3.6. Let $G$ be of type $G_{2}$ and $p \neq 3$. Then $F$ acts trivially on $W$ and the induced automorphism $\gamma: W \rightarrow W$ is the identity. Consequently, $\operatorname{Irr}(W)=$ $\operatorname{Irr}(W)^{\gamma}$. By [15, Table 22.2.6], there are 5 unipotent classes of $G$, which are all $F$-stable. Let $C$ be the unipotent class denoted by $G_{2}(a)$; we have $A(u) \cong \mathfrak{S}_{3}$ for $u \in C$. The set $C^{F}$ splits into three classes in $G^{F}$, with centraliser orders $6 q^{4}, 2 q^{4}, 3 q^{4}$. Thus, up to conjugation by elements in $G^{F}$, there is a unique $u_{0} \in C^{F}$ such that $\left|C_{G}\left(u_{0}\right)^{F}\right|=6 q^{4}$ and $F$ acts trivially on $A\left(u_{0}\right)$. This whole discussion also works for the Frobenius map $F_{p}$. Thus, we can even assume that $F_{p}\left(u_{0}\right)=u_{0}$ and $F_{p}$ acts trivially on $A\left(u_{0}\right)$. If $C^{\prime}$ is a unipotent class different from $C$, then $\left|A\left(u^{\prime}\right)\right| \leqslant 2$ for $u^{\prime} \in C$. Consequently, condition (\$) holds for all unipotent classes of $G$. The Springer correspondence is explicitly described by Spaltenstein [41, p. 329]; see Table 2, As in Example 2.7, we run the function ICCTable which yields the coefficients $p_{E^{\prime}, E}$. By inspection of Table 2, we see that there is just one case which is not covered by the arguments in Remark 3.4. The relevant unipotent class is the above-mentioned class $C$; we have

$$
\iota_{G}\left(E_{2,1}\right)=\left(C, \overline{\mathbb{Q}}_{\ell}\right) \quad \text { and } \quad \iota_{G}\left(E_{1,3}^{\prime}\right)=(C, \mathscr{E}) \quad \text { where } \quad \operatorname{dim} \mathscr{E}_{u}=2 .
$$

Using the output of ICCTable and the argument in Remark 3.4(a), we already see that $\delta_{E_{2,1}}=1$. However, we have $\left\langle Y_{E_{2,1}}, Y_{E_{1,3}^{\prime}}\right\rangle=0$ and so we can not apply the method in Remark 3.4(b). We now argue as follows. Using the output of ICCTable (see Table 2), we compute the coefficients $\tilde{p}_{E^{\prime}}$ in Remark 2.8. We obtain the following formula:

$$
Q_{1}\left(u_{0}\right)=(2 q+1) Y_{E_{2,1}}\left(u_{0}\right)+q Y_{E_{1,3}^{\prime}}\left(u_{0}\right)=(2 q+1)+2 q \delta_{E_{1,3}^{\prime}} .
$$

Thus, depending on whether $\delta_{E_{1,3}^{\prime}}$ equals +1 or -1 , we have $Q_{1}\left(u_{0}\right)=4 q+1$ or $Q_{1}\left(u_{0}\right)=1$. On the other hand, by Remark 2.8, $Q_{1}\left(u_{0}\right)$ also is the value at $u_{0}$ of the character of the permutation representation of $G^{F}$ on the cosets of $B_{0}^{F}$. We use this interpretation to show that $Q_{1}\left(u_{0}\right)>1$. For this purpose, it will be sufficient to show that $C_{G}\left(u_{0}\right)^{F} \nsubseteq B_{0}^{F}$. Assume, if possible, that $C_{G}\left(u_{0}\right)^{F} \subseteq B_{0}^{F}$. Then we also have $C_{G}\left(u_{0}\right)^{F_{p}} \subseteq B_{0}^{F_{p}}$. We have a natural homomorphism $B_{0}^{F_{p}} \rightarrow T_{0}^{F_{p}}$, with 
kernel consisting of unipotent elements only. If $p=2$, then $T_{0}^{F_{p}}=\{1\}$ and so $C_{G}\left(u_{0}\right)^{F_{p}}$ would be a unipotent group, contradiction to the fact that $A\left(u_{0}\right) \cong \mathfrak{S}_{3}$ is a quotient of $C_{G}\left(u_{0}\right)^{F_{p}}$. If $p \neq 2$ (and $p \neq 3$ ), then $A\left(u_{0}\right) \cong \mathfrak{S}_{3}$ will still be a quotient of the image of $C_{G}\left(u_{0}\right)^{F_{p}}$ in $T_{0}^{F_{p}}$, contradiction since $T_{0}$ is abelian. Thus, we do have $C_{G}\left(u_{0}\right)^{F} \nsubseteq B_{0}^{F}$ and so $Q_{1}\left(u_{0}\right)>1$, as claimed. But this forces $\delta_{E_{1,3}^{\prime}}=1$.

Remark 3.7. Assume that $\tilde{\gamma}=\operatorname{id}_{G}$ and $F=F_{p}^{m}$ where $m \geqslant 1$. Let us first consider the case where $m=1$ and $G^{F_{p}}=G\left(\mathbb{F}_{p}\right)$ is an untwisted Chevalley group over the prime field $\mathbb{F}_{p}$. The whole discussion above applies, of course, with $F_{p}$ instead of $F$. In order to have a separate notation from the general case, we introduce a superscript "\#” to various objects considered earlier. Thus, for $w \in W$, we denote by $R_{w}^{\sharp}$ the virtual representation of $G^{F_{p}}$ defined by Deligne-Lusztig, and by $Q_{w}^{\sharp}$ the corresponding Green function. For $E \in \operatorname{Irr}(W)$, let $R_{E}^{\sharp}: G^{F_{p}} \rightarrow \overline{\mathbb{Q}}_{\ell}$ be the corresponding almost character. (Note that, now, $F_{p}$ acts trivially on $W$ and so $\sigma_{E}=\operatorname{id}_{E}: E \rightarrow E$.) As in Remark 3.1, we assume that there exists an element $u_{0} \in C^{F_{p}}$ such that $F_{p}$ acts trivially on $A\left(u_{0}\right)$. By Remark [3.2, there is a welldefined sign, which we now denote by $\delta_{E}^{\sharp}= \pm 1$, such that

$$
R_{E}^{\sharp}\left(u_{0}\right)=\delta_{E}^{\sharp} p^{d_{E}} \operatorname{dim} \mathscr{E}_{u_{0}} .
$$

Having fixed the above notation, we now consider $F=F_{p}^{m}$ for any $m \geqslant 1$. Then we still have $F\left(u_{0}\right)=u_{0}$, and $F$ acts trivially on $A\left(u_{0}\right)$. Let $\delta_{E}= \pm 1$ be as in Remark 3.2, now with respect to $F$. Then, by [12, Theorem 3.7], we have

$$
\delta_{E}=\left(\delta_{E}^{\sharp}\right)^{m} ; \quad \text { in particular, } \delta_{E}=1 \text { whenever } m \text { is even. }
$$

Thus, in order to determine $\delta_{E}$, it is sufficient to consider the case where $m=1$. This will be our main tool in the discussion of groups of exceptional type, in order to deal with those cases which are not covered by Remark 3.4.

\section{Explicit Realisations of $G(q)$}

Assume that $G$ is a simple algebraic group. In order to perform explicit computations on a computer with elements of $G$ (as in the following sections), we need a concrete realisation of $G$ as a matrix group. Now, in principle it is well-known how to do this, using Chevalley's construction as explained in detail by Carter [2] and Steinberg [42. Computer programs are available as described by Cohen-MurrayTaylor [4], for example. Note that the starting point of this approach is the choice of a Chevalley basis in the corresponding simple Lie algebra over $\mathbb{C}$. For example, Mizuno [32, Table 12] explicitly specifies such a choice for type $E_{6}, E_{7}, E_{8}$. But this raises the following issue. If we want to perform computations with Mizuno's class representatives using the Cohen-Murray-Taylor programs, we would first need to clarify the relation between the chosen Chevalley bases - and the same issue arises with any other reference to the literature about explicit computations in $G$.

Here, we wish to advertise two recent developments with regard to these issues. Firstly, Lusztig [25] gives an explicit, canonical construction of $G$ as a matrix group, which does not depend at all on the choice of a Chevalley basis. Since this only yields root elements in $G$ for simple roots and their negatives, one still needs to specify a Chevalley basis for further computations. But then, secondly, [8] produces two canonical choices of a Chevalley basis, which differ from each other by a global sign and, thus, yield "canonical" root elements for all roots. 
The computer algebra package ChevLie [10] implements these constructions and works both in GAP4 [6] and Michel's version of GAP3 [30]. We briefly explain the constructions of [8], [25] and the basic functionality of the ChevLie package.

TABLE 3. Dynkin diagrams of simple Lie algebras

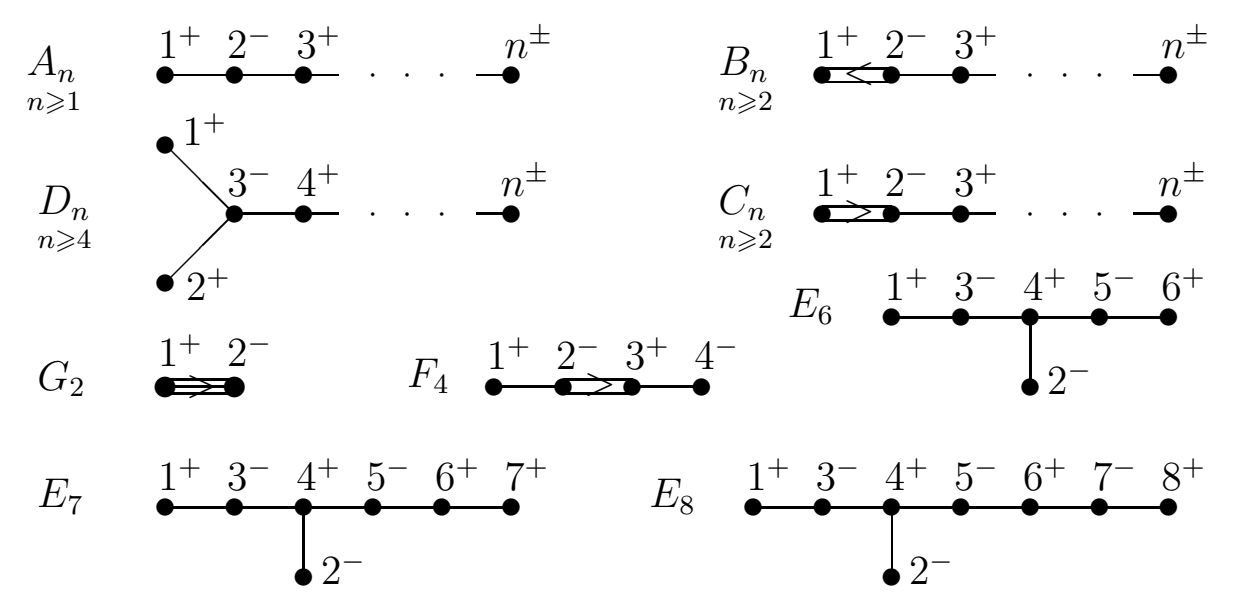

4.1. Cartan matrices and the $\epsilon$-function. Let $I$ be a finite index set and $A=\left(a_{i j}\right)_{i, j \in I}$ be the Cartan matrix of an irreducible (crystallographic) root system. In Table 3, we fix a labelling of the corresponding Dynkin diagram. Recall that $A$ can be recovered from the diagram as follows. For $i \in I$, we have $a_{i i}=2$. Now asssume that $i, j \in I$ are such that $i \neq j$. Then $a_{i j}=a_{j i}=0$ if $i, j$ are not joined by an edge. We have $a_{i j}=a_{j i}=1$ if $i, j$ are joined by a simple edge. Furthermore, $a_{i j}=-1$ and $a_{j i}=-2$ if $i, j$ are joined by a double edge with an arrow pointing towards $j$. Finally, $a_{i j}=-1$ and $a_{j i}=-3$ if $i, j$ are joined by a triple edge with an arrow pointing towards $j$. For example:

$$
B_{3}:\left(\begin{array}{rrr}
2 & -2 & 0 \\
-1 & 2 & -1 \\
0 & -1 & 2
\end{array}\right), \quad G_{2}:\left(\begin{array}{rr}
2 & -1 \\
-3 & 2
\end{array}\right) .
$$

In Table 3, we also specify a function $\epsilon: I \rightarrow\{ \pm 1\}$ such that $\epsilon(i)=-\epsilon(j)$ whenever $i \neq j$ and $a_{i j} \neq 0$. Note that, since the diagram is connected, there are exactly two such functions: if $\epsilon$ is one of them, then the other one is $-\epsilon$.

4.2. The Weyl group and the root system. Let $V$ be a $\mathbb{Q}$-vector space with a basis $\left\{\alpha_{i} \mid i \in I\right\}$. For $i \in I$, we define a linear map $s_{i}: V \rightarrow V$ by $s_{i}\left(\alpha_{j}\right):=$ $\alpha_{j}-a_{i j} \alpha_{i}$ for $j \in I$. Then $s_{i}^{2}=\mathrm{id}_{V}$ and so $s_{i} \in \mathrm{GL}(V)$. Then the corresponding Weyl group is given by $W:=\left\langle s_{i} \mid i \in I\right\rangle \subseteq \mathrm{GL}(V)$, with root system

$$
\Phi:=\left\{w\left(\alpha_{i}\right) \mid i \in I, w \in W\right\} \subseteq V,
$$

where $\left\{\alpha_{i} \mid i \in I\right\}$ is a system of simple roots. In CHEVIE [13], all of the above is realised by the function CoxeterGroup, which returns a record containing basic data corresponding to a given Dynkin diagram. For ChevLie, we essentially copied the code of that function, so that it works both in GAP3 and GAP4. Example:

$$
\begin{aligned}
& \text { gap> W:= WeylRecord ("B",2); } \\
& \text { gap> W.cartan \# the Cartan matrix }
\end{aligned}
$$




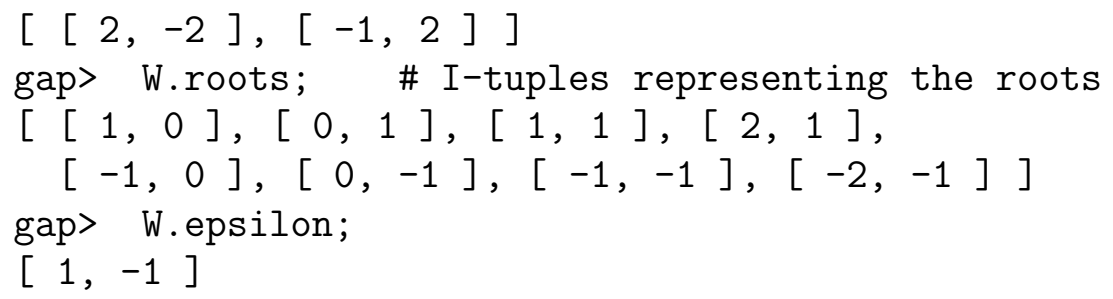

The record component epsilon holds the function $\epsilon: I \rightarrow\{ \pm 1\}$. (This is not present in the original CHEVIE system.)

4.3. The operators $e_{i}$ and $f_{i}$. For any $\alpha, \beta \in \Phi$ such that $\alpha \neq \pm \beta$, we define

$$
p_{\alpha, \beta}:=\max \{i \geqslant 0 \mid \beta+i \alpha \in \Phi\} \quad \text { and } \quad q_{\alpha, \beta}:=\max \{i \geqslant 0 \mid \beta-i \alpha \in \Phi\} .
$$

Thus, $\beta-q_{\alpha, \beta} \alpha, \ldots, \beta-\alpha, \beta, \beta+\alpha, \ldots, \beta+p_{\alpha, \beta} \alpha$ is the $\alpha$-string through $\beta$. Following Lusztig [25, §2], we now consider a $\mathbb{Q}$-vector space $M$ with a basis $\left\{u_{i} \mid i \in I\right\} \cup\left\{v_{\alpha} \mid \alpha \in \Phi\right\}$ and define linear maps $e_{i}: M \rightarrow M$ and $f_{i}: M \rightarrow M$ by the following formulae, where $j \in I$ and $\alpha \in \Phi$.

$$
\begin{aligned}
& e_{i}\left(u_{j}\right):=\left|a_{j i}\right| v_{\alpha_{i}}, \quad e_{i}\left(v_{\alpha}\right):=\left\{\begin{array}{cl}
\left(q_{\alpha_{i}, \alpha}+1\right) v_{\alpha+\alpha_{i}} & \text { if } \alpha+\alpha_{i} \in \Phi, \\
u_{i} & \text { if } \alpha=-\alpha_{i}, \\
0 & \text { otherwise, }
\end{array}\right. \\
& f_{i}\left(u_{j}\right):=\left|a_{j i}\right| v_{-\alpha_{i}}, \quad f_{i}\left(v_{\alpha}\right):=\left\{\begin{array}{cl}
\left(p_{\alpha_{i}, \alpha}+1\right) v_{\alpha-\alpha_{i}} & \text { if } \alpha-\alpha_{i} \in \Phi, \\
u_{i} & \text { if } \alpha=\alpha_{i}, \\
0 & \text { otherwise. }
\end{array}\right.
\end{aligned}
$$

Note that all entries of the matrices of $e_{i}, f_{i}$ with respect to the given basis of $M$ are non-negative integers. We consider $\operatorname{End}(M)$ as a Lie algebra with the usual Lie bracket $[x, y]:=x \circ y-y \circ x$ for $x, y \in \operatorname{End}(M)$. We set $h_{i}:=\left[e_{i}, f_{i}\right]$ for $i \in I$. As in [8, §4], consider the Lie subalgebra $\mathfrak{g} \subseteq \operatorname{End}(M)$ generated by $e_{i}, f_{i}(i \in I)$. Then $\mathfrak{g}$ is a (split) simple Lie algebra with Cartan subalgebra $\mathfrak{h}:=\left\langle h_{i} \mid i \in I\right\rangle_{\mathbb{Q}}$ and corresponding root system $\Phi$. In particular, we have the Cartan decomposition

$$
\mathfrak{g}=\mathfrak{h} \oplus \bigoplus_{\alpha \in \Phi} \mathfrak{g}_{\alpha} \quad \text { where } \quad \operatorname{dim} \mathfrak{g}_{\alpha}=1 \text { for all } \alpha \in \Phi .
$$

In ChevLie, we obtain matrices representing $e_{i}, f_{i}$ through the following command.

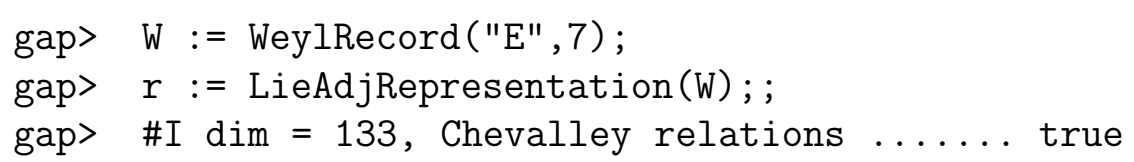

If the basis vectors $\left\{u_{i} \mid i \in I\right\} \cup\left\{v_{\alpha} \mid \alpha \in \Phi\right\}$ are ordered as in [8, Lemma 4.1], then each $e_{i}$ is a nilpotent upper triangular matrix and each $f_{i}$ is a nilpotent lower triangular matrix. This convention is used in ChevLie. There is also the function LieMinusculeRepresentation which constructs the matrices in a representation with a minuscule heighest weight, as in [9].

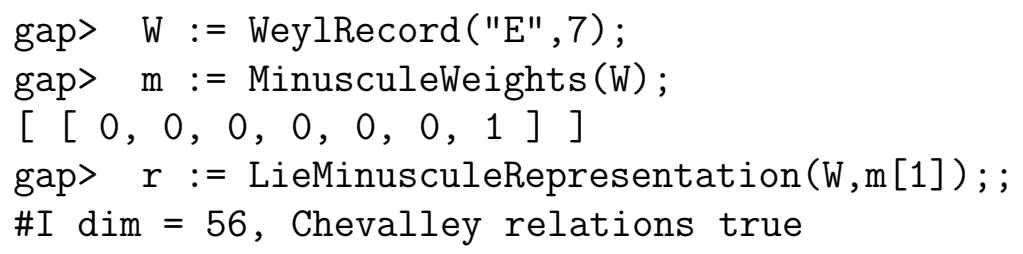


4.4. Lusztig's construction of Chevalley groups. Following Lusztig [25, §2], we now obtain a Chevalley group over any field as follows. Since the $e_{i}$ and $f_{i}$ are nilpotent, we can define $x_{i}(t):=\exp \left(t e_{i}\right) \in \mathrm{GL}(M)$ and $y_{i}(t):=\exp \left(t f_{i}\right) \in \mathrm{GL}(M)$ for all $i \in I$ and $t \in \mathbb{Q}$. Explicitly, we have:

$$
\begin{gathered}
x_{i}(t)\left(u_{j}\right)=u_{j}+\left|a_{j i}\right| t v_{\alpha_{i}}, \quad x_{i}(t)\left(v_{-\alpha_{i}}\right)=v_{-\alpha_{i}}+t u_{i}+t^{2} v_{\alpha_{i}}, \\
x_{i}(t)\left(v_{\alpha_{i}}\right)=v_{\alpha_{i}}, \quad x_{i}(t)\left(v_{\alpha}\right)=\sum_{k \geqslant 0, \alpha+k \alpha_{i} \in \Phi}\left(\begin{array}{c}
k+q_{\alpha_{i}, \alpha} \\
k
\end{array}\right) t^{k} v_{\alpha+k \alpha_{i}}, \\
y_{i}(t)\left(u_{j}\right)=u_{j}+\left|a_{j i}\right| t v_{-\alpha_{i}}, \quad y_{i}(t)\left(v_{\alpha_{i}}\right)=v_{\alpha_{i}}+t u_{i}+t^{2} v_{-\alpha_{i}}, \\
y_{i}(t)\left(v_{-\alpha_{i}}\right)=v_{-\alpha_{i}}, \quad y_{i}(t)\left(v_{\alpha}\right)=\sum_{k \geqslant 0, \alpha-k \alpha_{i} \in \Phi}\left(\begin{array}{c}
k+p_{\alpha_{i}, \alpha} \\
k
\end{array}\right) t^{k} v_{\alpha-k \alpha_{i}},
\end{gathered}
$$

where $j \in I$ and $\alpha \in \Phi, \alpha \neq \pm \alpha_{i}$. (Compare with the formulae in [2, §4.3].) Now let $K$ be any field and $\bar{M}$ be a $K$-vector space with a basis $\left\{\bar{u}_{i} \mid i \in I\right\} \cup\left\{\bar{v}_{\alpha} \mid \alpha \in \Phi\right\}$. For $i \in I$ and $t \in K$, we define $\bar{x}_{i}(t) \in \mathrm{GL}(\bar{M})$ and $\bar{y}_{i}(t) \in \mathrm{GL}(\bar{M})$ by formulae as above (which involve only integer coefficients; see also [2, §4.4].) Then

$$
G_{K}:=\left\langle\bar{x}_{i}(t), \bar{y}_{i}(t) \mid i \in I, t \in K\right\rangle \subseteq \mathrm{GL}(\bar{M})
$$

is a Chevalley group over $K$.

4.5. The $\epsilon$-canonical Chevalley basis. For each $\alpha \in \Phi$, let us choose a non-zero element $e_{\alpha} \in \mathfrak{g}_{\alpha}$. If $\alpha, \beta \in \Phi$ are such that $\alpha+\beta \in \Phi$, then we define $N_{\alpha, \beta} \in \mathbb{Q}$ by $\left[e_{\alpha}, e_{\beta}\right]=N_{\alpha, \beta} e_{\alpha+\beta}$. Now $\left\{e_{\alpha} \mid \alpha \in \Phi\right\}$ is called a Chevalley basis if

$$
N_{\alpha, \beta}= \pm\left(q_{\alpha, \beta}+1\right) \quad \text { for all } \alpha, \beta \in \Phi \text { such that } \alpha+\beta \in \Phi \text {. }
$$

Clearly, if $\left\{e_{\alpha} \mid \alpha \in \Phi\right\}$ is a Chevalley basis, then so is $\left\{ \pm e_{\alpha} \mid \alpha \in \Phi\right\}$, for any choice of the signs. Now, having fixed $\epsilon: I \rightarrow\{ \pm 1\}$, there is a unique Chevalley basis $\left\{\mathbf{e}_{\alpha}^{\epsilon} \mid \alpha \in \Phi\right\}$ such that the following relations hold, for any $i \in I$ :

$$
\begin{array}{rlrl}
\mathbf{e}_{\alpha_{i}}^{\epsilon} & =\epsilon(i) e_{i}, & \mathbf{e}_{-\alpha_{i}}^{\epsilon}=-\epsilon(i) f_{i}, \\
{\left[e_{i}, \mathbf{e}_{\alpha}^{\epsilon}\right]} & =\left(q_{\alpha_{i}, \alpha}+1\right) \mathbf{e}_{\alpha+\alpha_{i}}^{\epsilon} & & \text { if } \alpha+\alpha_{i} \in \Phi, \\
{\left[f_{i}, \mathbf{e}_{\alpha}^{\epsilon}\right]} & =\left(p_{\alpha_{i}, \alpha}+1\right) \mathbf{e}_{\alpha-\alpha_{i}}^{\epsilon} & \text { if } \alpha-\alpha_{i} \in \Phi .
\end{array}
$$

(See [8, Theorem 5.7 and Example 5.9].) If we replace $\epsilon$ by $-\epsilon$, then $\mathbf{e}_{\alpha}^{-\epsilon}=-\mathbf{e}_{\alpha}^{\epsilon}$ for all $\alpha \in \Phi$. In ChevLie, the complete list of elements $\left\{\mathbf{e}_{\alpha}^{\epsilon} \mid \alpha \in \Phi\right\}$, as matrices with respect to the basis $\left\{u_{i} \mid i \in I\right\} \cup\left\{v_{\alpha} \mid \alpha \in \Phi\right\}$ of $M$, is obtained through the command CanonicalChevalleyBasis (W).

4.6. Root elements. Let $\alpha \in \Phi$. By [8, Cor. 5.6], the linear map $\mathbf{e}_{\alpha}^{\epsilon} \in \operatorname{End}(M)$ is nilpotent and so we can define $x_{\alpha}^{\epsilon}(t):=\exp \left(t \mathbf{e}_{\alpha}^{\epsilon}\right) \in \mathrm{GL}(M)$ for any $t \in \mathbb{Q}$. As in $\$ 4.4$, if $K$ is any field, then we obtain analogous elements $\bar{x}_{\alpha}^{\epsilon}(t) \in G_{K}$ for $t \in K$. If $\epsilon$ is replaced by $-\epsilon$, then $\bar{x}_{\alpha}^{-\epsilon}(t)=\bar{x}_{\alpha}^{\epsilon}(-t)$ for all $t \in K$. Thus, having fixed $\epsilon$, we obtain "canonical" root elements $\bar{x}_{\alpha}^{\epsilon}(t) \in G_{K}$. In ChevLie, these are obtained as follows.

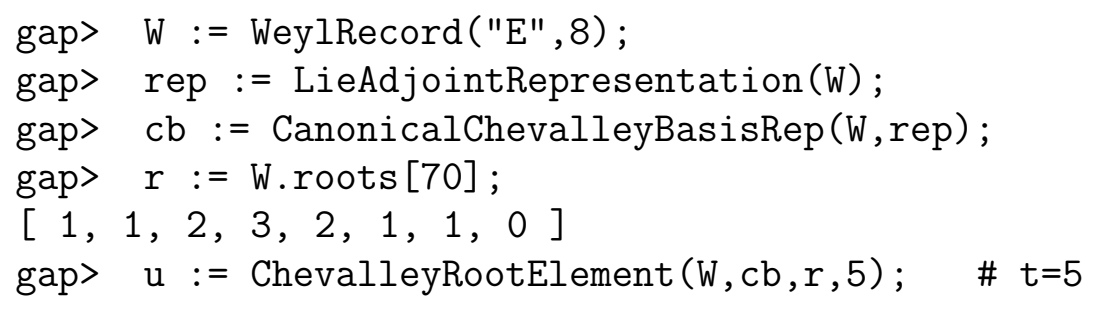


< matrix 248x248 over the integers >

(One can equally well use elements from finite fields, of course; furthermore, instead of the adjoint representation, one can also use a representation with a minuscule highest weight, if such a representation exists.) Once the elements $\bar{x}_{\alpha}^{\epsilon}(t) \in G_{K}$ are available, we can also define elements $\bar{h}_{\alpha}^{\epsilon}(t) \in G_{K}$ and $\bar{n}_{\alpha}^{\epsilon}(t) \in G_{K}$ by analogous formulae as in [2, Lemma 6.4.4]. These elements yield the familiar diagonal elements and lifts of reflections in the Weyl group, respectively.

In ChevLie, the basis of $M$ is ordered such that all $\bar{x}_{\alpha}^{\epsilon}(t), \alpha \in \Phi^{+}$, are represented by unipotent upper triangular matrices, and all $\bar{x}_{\alpha}^{\epsilon}(t), \alpha \in \Phi^{-}$, by unipotent lower triangular matrices; futhermore, all $\bar{h}_{\alpha}^{\epsilon}(t)$ are diagonal and all $\bar{n}_{\alpha}^{\epsilon}(t)$ are monomial matrices. Thus, the unipotent radical of the standard Borel subgroup of $G_{K}$, that is, the subgroup

$$
U_{K}=\left\langle\bar{x}_{\alpha}^{\epsilon}(t) \mid \alpha \in \Phi^{+}, t \in K\right\rangle \subseteq G_{K}
$$

consists precisely of the unipotent upper triangular matrices in $G_{K}$.

4.7. Computing estimates for $Q_{1}(u)$. Now let $G=G_{k}$, where $k=\overline{\mathbb{F}}_{p}$ as in the previous sections, and $F_{p}: G \rightarrow G$ is given by $F_{p}\left(\bar{x}_{\alpha}^{\epsilon}(t)\right)=\bar{x}_{\alpha}^{\epsilon}\left(t^{p}\right)$ for $\alpha \in \Phi$ and $t \in k$. Once the above functions are available, it is straightforward to write a program which computes the cardinalities of the sets $Q_{1, w}(u)$ in Lemma 2.9, where $u \in G^{F_{p}}$. For $w \in W$, let $\Phi_{w}^{+}=\left\{\beta_{1}, \ldots, \beta_{l}\right\}$ where $l=l(w)$. Then

$$
U_{w}^{F_{p}}=\left\{\bar{x}_{\beta_{1}}^{\epsilon}\left(t_{1}\right) \cdots \bar{x}_{\beta_{l}}^{\epsilon}\left(t_{l}\right) \mid t_{i} \in \mathbb{F}_{p}\right\},
$$

with uniqueness of expression. Thus, by running systematically over all tuples $\left(t_{1}, \ldots, t_{l}\right) \in \mathbb{F}_{p}^{l}$, we have a way of running through the elements of $U_{w}^{F_{p}}$, one by one. For each $v \in U_{w}^{F_{p}}$, we need to check if $v^{\prime}:=\dot{w}^{-1} v^{-1} u v \dot{w} \in B_{0}^{F_{p}}$ which, by the remarks in \$4.6, is simply done by testing if $v^{\prime}$ is an upper triangular matrix. (Some modifications are needed for twisted groups; see \$7.1 below.)

This description shows that computer memory is not an issue, but speed is critical. We shall have to perform several millions of multiplications of matrices (of moderate size) over small finite fields. For this purpose, the GAP [6] function ImmutableMatrix turns out to be particularly efficient. It converts a given matrix into an internal format which appears to be highly optimized concerning space and runtime.

\section{On the GREen FUnCtions OF TYPE $F_{4}$ IN CHARACTERISTIC 3}

Throughout this section, let $G$ be a simple algebraic group of type $F_{4}$. We have $G=\left\langle x_{\alpha}(t) \mid \alpha \in \Phi, t \in k\right\rangle$ where $\Phi$ is the root system of $G$ with respect to $T_{0}$. Let $\left\{\alpha_{1}, \alpha_{2}, \alpha_{3}, \alpha_{4}\right\}$ be the set of simple roots with respect to $B$, where the labelling is as in Table 3. We assume that $G$ is defined and split over $\mathbb{F}_{p}$, with corresponding Frobenius map $F_{p}: G \rightarrow G$ such that $F_{p}(t)=t^{p}$ for all $t \in T_{0}$. Let $F=F_{p}^{m}$ where $m \geqslant 1$. Then

$$
G^{F}=F_{4}(q) \quad \text { where } \quad q=p^{m} .
$$

For $p>3$, the Green functions of $G^{F}$ have been determined by Shoji [35]. For $p=$ 2, the Green functions are explicitly computed by Malle [28]. It is briefly remarked by Marcelo-Shinoda [29] that Shoji's computations remain valid for $p=3$. Since further details are omitted in [29], we provide here an independent verification based on the results in Section 3, this will also serve as a model for the later case 
studies in Sections [6 9]. In the following, if $\alpha=\sum_{i=1}^{4} n_{i} \alpha_{i} \in \Phi$, we just write $x_{n_{1} n_{2} n_{3} n_{4}}(t)$ instead of $x_{\alpha}(t)$.

5.1. Critical unipotent classes for $p=3$. Assume from now on that $p=3$. We have $|\operatorname{Irr}(W)|=25$ and the character table of $W$ is available in CHEVIE. Now $F$ acts trivially on $W$ and $\gamma: W \rightarrow W$ is the identity. Consequently, $\operatorname{Irr}(W)=$ $\operatorname{Irr}(W)^{\gamma}$. By Shoji [34, there are 16 unipotent classes of $G$, which are all $F_{3}$-stable. Furthermore, for each unipotent class $C$, there exists an element $u_{0} \in C$ such that $F_{3}\left(u_{0}\right)=u_{0}$ and $F_{3}$ acts trivially on $A\left(u_{0}\right)$; see [34, Table 6]. Thus, condition (\$) in Section 3 holds. The Springer correspondence is explicitly described by Spaltenstein [41, p. 330]. As in Example 2.7, we run the function ICCTable which yields the coefficients $p_{E^{\prime}, E}$. By inspection of the output, we see that $p_{E^{\prime}, E_{1}} \in\{0,1\}$ for all $E^{\prime} \in \operatorname{Irr}(W)$, where $E_{1}$ is the trivial representation of $W$. Hence, by the argument in Remark 3.4(a), we already have that

$$
\delta_{E_{0}}=1 \quad \text { for all } E_{0} \in \operatorname{Irr}(W) \text { such that } \iota_{G}\left(E_{0}\right)=\left(C, \overline{\mathbb{Q}}_{\ell}\right) .
$$

There are further cases which are not covered by the arguments in Remark 3.4(b); these are specified in Table 4. The last two columns specify $E, E_{0} \in \operatorname{Irr}(W)$ such that $\iota_{G}\left(E_{0}\right)=\left(C, \overline{\mathbb{Q}}_{\ell}\right)$ and $\iota_{G}(E)=(C, \mathscr{E})$ with $\mathscr{E} \neq \overline{\mathbb{Q}}_{\ell}$.

TABLE 4. Critical unipotent classes for type $F_{4}$ with $p=3$

\begin{tabular}{cccccc}
\hline$C$ & $\operatorname{dim} C_{G}(u)$ & $A(u)$ & $\mid C_{G}(u)^{F}$ & $E_{0}$ & $E: \operatorname{dim} \mathscr{E}_{u}$ \\
\hline$F_{4}\left(a_{1}\right)$ & 6 & $\mathbb{Z} / 2 \mathbb{Z}$ & $2 q^{6}, 2 q^{6}$ & $\chi_{4,1}$ & $\chi_{2,3}: 1$ \\
\hline$F_{4}\left(a_{2}\right)$ & 8 & $\mathbb{Z} / 2 \mathbb{Z}$ & $2 q^{8}, 2 q^{8}$ & $\chi_{9,1}$ & $\chi_{2,1}: 1$ \\
\hline \multirow{2}{*}{$F_{4}\left(a_{3}\right)$} & 12 & $\mathfrak{S}_{4}$ & $24 q^{12}, 8 q^{12}, 4 q^{12}, 4 q^{12}, 3 q^{12}$ & $\chi_{12}$ & $\chi_{9,3}: 3$ \\
& & & & & $\chi_{1,3}: 3$ \\
\hline$C_{3}\left(a_{1}\right)$ & 14 & $\mathbb{Z} / 2 \mathbb{Z}$ & $2 q^{12}\left(q^{2}-1\right), 2 q^{12}\left(q^{2}-1\right)$ & $\chi_{16}$ & $\chi_{4,3}: 1$ \\
\hline
\end{tabular}

In the table, we use the notation of Spaltenstein [41] for $\operatorname{Irr}(W)$. The translation to the notation of Carter [3, §13.2] (or CHEVIE) is as follows.

\begin{tabular}{ccccccccc}
\hline$\chi_{4,1}$ & $\chi_{2,3}$ & $\chi_{9,1}$ & $\chi_{2,1}$ & $\chi_{12}$ & $\chi_{9,3}$ & $\chi_{6,2}$ & $\chi_{1,3}$ & $\chi_{4,3}$ \\
\hline$\phi_{4,8}$ & $\phi_{2,4}^{\prime}$ & $\phi_{9,2}$ & $\phi_{2,4}^{\prime \prime}$ & $\phi_{12,4}$ & $\phi_{9,6}^{\prime}$ & $\phi_{6,6}^{\prime \prime}$ & $\phi_{1,12}^{\prime}$ & $\phi_{4,7}^{\prime \prime}$ \\
\hline
\end{tabular}

5.2. The class $F_{4}\left(a_{1}\right)$. Let $C$ be the unipotent class denoted by $F_{4}\left(a_{1}\right)$. Since $A(u) \cong \mathbb{Z} / 2 \mathbb{Z}$ for $u \in C$, we are in the situation of Example 3.5, with $E_{0}=\chi_{4,1}$ and $E=\chi_{2,3}$; see Table 4. We already know that there exists some $u_{0} \in C^{F}$ such that $F$ acts trivially on $A\left(u_{0}\right)$ and $\delta_{\chi_{4,1}}=\delta_{\chi_{2,3}}=1$. The only remaining problem is to identify $u_{0}$ in a given list of class representatives. For a certain choice of a Chevalley basis in the Lie algebra of $G$, a representative $\tilde{u} \in C$ is explicitly described by Lawther [14, Table A]. Since our "canonical" Chevalley basis in Section 4 may be different from that in [14], we can only say that

$$
\tilde{u}:=x_{1000}\left(\varepsilon_{1}\right) x_{0100}\left(\varepsilon_{2}\right) x_{0110}\left(\varepsilon_{3}\right) x_{0011}\left(\varepsilon_{4}\right) \in C
$$

where $\varepsilon_{i}= \pm 1$ for $1 \leqslant i \leqslant 4$. Clearly, we have $\tilde{u} \in G^{F_{3}}$. Using our computer programs in $\$ 4$, we check explicitly that all elements $\tilde{u}$ as above, for all possible choices of the $\varepsilon_{i}$, are conjugate under elements of $T_{0}^{F_{3}}$. Hence, we may assume without loss of generality that $\varepsilon_{i}=1$ for all $i$. Now consider the signs $\delta_{\chi_{4,1}}$ and 
$\delta_{\chi_{2,3}}$ with respect to $\tilde{u}$. Since $\iota_{G}\left(\chi_{4,1}\right)=\left(C, \overline{\mathbb{Q}}_{\ell}\right)$, we already know that $\delta_{\chi_{4,1}}=1$. We claim that we also have $\delta_{\chi_{2,3}}=1$. Since $\tilde{u} \in C^{F_{3}}$, we can apply Remark 3.7. Thus, it will be sufficient to determine $\delta_{\chi_{2,3}}$ in the special case where $m=1$. We now argue as in Example 3.6. Using the output of ICCTable, we find the coefficients $\tilde{p}_{E^{\prime}}$ in $\$ 2.8$. This yields the formula

$$
Q_{1}(\tilde{u})=(4 q+1) Y_{\chi_{4,1}}(\tilde{u})+2 q Y_{\chi_{2,3}}(\tilde{u})=(4 q+1)+2 q \delta_{\chi_{2,3}} .
$$

Setting $q=3$, we obtain $Q_{1}(\tilde{u})=13+6 \delta_{\chi_{2,3}} \in\{19,7\}$. On the other hand, by Remark 2.10, we can try to directly compute the value of $Q_{1}(\tilde{u})$ (or, at least, a lower bound for that value), by running through the sets $Q_{1, w}(\tilde{u})$ and checking if the corresponding coset representatives are fixed by $\tilde{u}$. It turns out that we just need to go up to $l(w) \leqslant 3$ in order to find 19 cosets that are fixed. (Since we already know that $Q_{1}(\tilde{u}) \in\{19,7\}$, it would actually be enough to find strictly more than 7 cosets that are fixed - this simple remark will be important in later sections when the values of $Q_{1}$ get significantly larger.) Thus, we do have $Q_{1}(\tilde{u})=19$ and, indeed, $\tilde{u} \in C$ is a representative such that $\delta_{\chi_{4,1}}=\delta_{\chi_{2,3}}=1$ (regardless of the choice of a Chevalley basis).

5.3. The class $F_{4}\left(a_{2}\right)$. Let $C$ be the unipotent class denoted by $F_{4}\left(a_{2}\right)$. Again, we are in the situation of Example 3.5, now with $E_{0}=\chi_{9,1}$ and $E=\chi_{2,1}$; see Table 4 . As in the previous case, there exists some $u_{0} \in C^{F}$ such that $\delta_{\chi_{9,1}}=\delta_{\chi_{2,1}}=1$. The only remaining problem is to identify $u_{0}$ in a given list of class representatives. By Lawther [14, Table A], there is a choice of signs $\varepsilon_{i}= \pm 1$ such that

$$
\tilde{u}:=x_{1100}\left(\varepsilon_{1}\right) x_{0120}\left(\varepsilon_{2}\right) x_{0001}\left(\varepsilon_{3}\right) x_{0011}\left(\varepsilon_{4}\right) \in C .
$$

We check again that all elements as above, for all possible choices of the signs, are conjugate under elements of $T_{0}^{F_{3}}$. Hence, as in the previous case, we may assume without loss of generality that $\varepsilon_{i}=1$ for all $i$. We consider the signs $\delta_{\chi_{9,1}}$ and $\delta_{\chi_{2,1}}$ with respect to $\tilde{u}$. Since $\iota_{G}\left(\chi_{9,1}\right)=\left(C, \overline{\mathbb{Q}}_{\ell}\right)$, we already know that $\delta_{\chi_{9,1}}=1$. Since $\tilde{u} \in C^{F_{3}}$, it will again be sufficient to determine $\delta_{\chi_{2,1}}$ in the special case where $m=1$. Using the output of ICCTable, we find the formula

$$
Q_{1}(\tilde{u})=\left(9 q^{2}+4 q+1\right) Y_{\chi_{9,1}}(\tilde{u})+2 q^{2} Y_{\chi_{2,1}}(\tilde{u})=\left(9 q^{2}+4 q+1\right)+2 q^{2} \delta_{\chi_{2,1}}
$$

Setting $q=3$, we obtain $Q_{1}(\tilde{u})=94+18 \delta_{\chi_{2,1}} \in\{112,76\}$. Again, by an explicit computation counting coset representatives, we find that $Q_{1}(\tilde{u})=112$. (We just need to look at sets $Q_{1, w}(\tilde{u})$ where $l(w) \leqslant 7$.) Thus, indeed, $\tilde{u} \in C$ is a representative such that $\delta_{\chi_{9,1}}=\delta_{\chi_{2,1}}=1$ (regardless of the choice of a Chevalley basis).

5.4. The class $F_{4}\left(a_{3}\right)$. Let $C$ be the unipotent class denoted by $F_{4}\left(a_{3}\right)$. We have $A(u) \cong \mathfrak{S}_{4}$ for $u \in C$. By Shoji [34, Table 6], the set $C^{F}$ splits into five classes in $G^{F}$, with centraliser orders $24 q^{12}, 4 q^{12}, 8 q^{12}, 3 q^{12}, 4 q^{12}$. Thus, up to conjugation by elements in $G^{F}$, there is a unique $u_{0} \in C^{F}$ such that $\left|C_{G}\left(u_{0}\right)^{F}\right|=24 q^{12}$ and $F$ acts trivially on $A\left(u_{0}\right)$. Now, via the Springer correspondence, there are four irreducible representations of $W$ associated with $C$. These are $\chi_{12}, \chi_{9,3}, \chi_{6,2}, \chi_{1,3}$; see Table 4. We already know that $\delta_{\chi_{12}}=1$. Now $u_{0}$ can be chosen to be fixed by $F_{3}$; see the explicit expression in [34, Table 6]. So, by Remark 3.7, it is sufficient to determine $\delta_{\chi_{6,2}}, \delta_{\chi_{9,3}}, \delta_{\chi_{2,1}}$ in the special case where $m=1$. 
Let $u_{0}=u_{1}, u_{2}, u_{3}, u_{4}, u_{5} \in C^{F_{3}}$ be representatives of the $G^{F_{3}}$-conjugacy classes that are contained in $C^{F_{3}}$, and let $a_{1}, a_{2}, a_{3}, a_{4}, a_{5} \in A\left(u_{0}\right)$ be corresponding representatives of the conjugacy classes of $A\left(u_{0}\right)$ (see Remark 3.1). Using the output of ICCTable, and setting $q=3$, we find the formula

$$
\begin{aligned}
Q_{1}\left(u_{i}\right)= & \left(12 q^{4}+16 q^{3}+9 q^{2}+4 q+1\right) Y_{\chi_{12}}\left(u_{i}\right) \\
& +\left(6 q^{4}+4 q^{3}\right) Y_{\chi_{6,2}}\left(u_{i}\right)+\left(9 q^{4}+8 q^{3}+2 q^{2}\right) Y_{\chi_{9,3}}\left(u_{i}\right)+q^{4} Y_{\chi_{1,3}}\left(u_{i}\right) \\
= & 1498 Y_{\chi_{12}}\left(u_{i}\right)+594 Y_{\chi_{6,2}}\left(u_{i}\right)+963 Y_{\chi_{9,3}}\left(u_{i}\right)+81 Y_{\chi_{1,3}}\left(u_{i}\right) \quad(q=3),
\end{aligned}
$$

for $1 \leqslant i \leqslant 5$. Now, up to the signs $\delta_{\chi_{6,2}}, \delta_{\chi_{9,3}}, \delta_{\chi_{1,3}}$, the values of the $Y$-functions on $u_{i}$ are given by character values of $\mathfrak{S}_{4}$; see Remark 3.2 . Thus, up to those signs, we can explicitly determine the values $Q_{1}\left(u_{i}\right)$. We find that $Q_{1}\left(u_{i}\right) \leqslant 5818$ for all $i$, regardless of what the signs $\delta_{\chi_{6,2}}, \delta_{\chi_{9,3}}, \delta_{\chi_{1,3}}$ are; furthermore,

$$
Q_{1}\left(u_{i}\right)=5818 \quad \Longleftrightarrow \quad i=1 \quad \text { and } \quad \delta_{\chi 6,2}=\delta_{\chi 9,3}=\delta_{\chi 1,3}=1 .
$$

Hence, if we can find an element $\tilde{u} \in C^{F_{3}}$ such that $Q_{1}(\tilde{u})=5818$, then $\tilde{u}$ must be conjugate to $u_{0}=u_{1}$ in $G^{F_{3}}$ and $\delta_{\chi_{6,2}}=\delta_{\chi_{9,3}}=\delta_{\chi_{1,3}}=1$. Now, using the list of representatives in [34, Table 6] and adjusting some signs, we consider the element

$$
\tilde{u}:=x_{1100}(1) x_{0120}(-1) x_{0122}(1) x_{1122}(-1) \in G^{F_{3}},
$$

where we work with the "canonical" Chevalley basis as in Section 4. We check that, in the adjoint representation, $\tilde{u}$ has Jordan blocks of sizes $7,6^{2}, 5^{3}, 3^{6}$. Hence, we have $\tilde{u} \in C$; see [14, Table 4]. By an explicit computation counting coset representatives, we find that $Q_{1}(\tilde{u})=5818$. (We just need to look at sets $Q_{1, w}(\tilde{u})$ where $l(w) \leqslant 9$.) Thus, indeed, $u_{0}, \tilde{u}$ are conjugate in $G^{F_{3}}$ and we do have $\delta_{\chi_{6,2}}=$ $\delta_{\chi_{9,3}}=\delta_{\chi_{1,3}}=1$.

5.5. The class $C_{3}\left(a_{1}\right)$. Let $C$ be the unipotent class denoted by $C_{3}\left(a_{1}\right)$. Again, we are in the situation of Example [3.5, now with $E_{0}=\chi_{16}$ and $E=\chi_{4,3}$; see Table 4. As in the first case, there exists some $u_{0} \in C^{F}$ such that $\delta_{\chi_{16}}=\delta_{\chi_{4,3}}=1$. The only remaining problem is to identify $u_{0}$ in a given list of class representatives. By Lawther [14, Table A], there is a choice of signs $\varepsilon_{i}= \pm 1$ such that

$$
x_{0100}\left(\varepsilon_{1}\right) x_{0001}\left(\varepsilon_{2}\right) x_{0120}\left(\varepsilon_{3}\right) \in C \quad\left(\text { where } \varepsilon_{i}= \pm 1\right) .
$$

Now we find that all elements as above, for all possible choices of the signs, are conjugate under elements of $T_{0}^{F_{3}}$ to one of the following two elements:

$$
\tilde{u}^{ \pm}:=x_{0100}(1) x_{0001}(1) x_{0120}( \pm 1) .
$$

We check that, in the adjoint representation, both $\tilde{u}^{+}$and $\tilde{u}^{-}$have Jordan blocks of sizes $7,6^{2}, 5,4^{4}, 3^{3}, 1^{3}$. Hence, we have $\tilde{u}^{ \pm} \in C$; see [14, Table 4]. We consider the signs $\delta_{\chi_{16}}^{ \pm}$and $\delta_{\chi_{4,3}}^{ \pm}$with respect to $\tilde{u}^{ \pm}$. Since $\iota_{G}\left(\chi_{16}\right)=\left(C, \overline{\mathbb{Q}}_{\ell}\right)$, we already know that $\delta_{\chi_{16}}^{ \pm}=1$. Since $\tilde{u}^{ \pm} \in C^{F_{3}}$, it will again be sufficient to determine $\delta_{\chi_{4,3}}^{ \pm}$in the special case where $m=1$. Using the output of ICCTable, we find the formula

$$
\begin{gathered}
Q_{1}\left(\tilde{u}^{ \pm}\right)=\left(16 q^{5}+36 q^{4}+28 q^{3}+11 q^{2}+4 q+1\right) Y_{\chi_{16}}\left(\tilde{u}^{ \pm}\right) \\
+\left(4 q^{5}+10 q^{4}+8 q^{3}+2 q^{2}\right) Y_{\chi_{4,3}}\left(\tilde{u}^{ \pm}\right) .
\end{gathered}
$$

Setting $q=3$, we obtain $Q_{1}\left(\tilde{u}^{ \pm}\right)=7672+2016 \delta_{\chi_{4,3}}^{ \pm} \in\{9688,5656\}$. By an explicit computation counting coset representatives, we find that $Q_{1}\left(\tilde{u}^{ \pm}\right)=9688$. (For $\tilde{u}^{+}$, we just need to look at sets $Q_{1, w}\left(\tilde{u}^{ \pm}\right)$where $l(w) \leqslant 13$ in order to find 9688 cosets 
that are fixed; for $\tilde{u}^{-}$we have to go up to $l(w) \leqslant 14$ in order to find strictly more than 5656 cosets that are fixed.) In particular, this shows that $\tilde{u}^{+}, \tilde{u}^{-}$are conjugate in $G^{F_{3}}$. Hence, indeed, $u=\tilde{u}^{+} \in C$ is a representative such that $\delta_{\chi_{16}}=\delta_{\chi_{4,3}}=1$ (independently of the choice of a Chevalley basis).

Remark 5.6. By analogous arguments, we obtain an independent verification of the results of Malle [28] on the Green functions of $F_{4}\left(2^{m}\right)$.

\section{On the Green functions of untwisted $E_{6}$ in Characteristic 3}

Throughout this section, let $G$ be a simple algebraic group of (adjoint) type $E_{6}$. We have $G=\left\langle x_{\alpha}(t) \mid \alpha \in \Phi, t \in k\right\rangle$ where $\Phi$ is the root system of $G$ with respect to $T_{0}$. Let $\left\{\alpha_{i} \mid 1 \leqslant i \leqslant 6\right\}$ be the set of simple roots with respect to $B_{0}$, where the labelling is chosen as in Table 3. We assume that $G$ is defined and split over $\mathbb{F}_{p}$, with corresponding Frobenius map $F_{p}: G \rightarrow G$ such that $F_{p}(t)=t^{p}$ for all $t \in T_{0}$. Let $F=F_{p}^{m}$ where $m \geqslant 1$. Then

$$
G^{F}=E_{6}(q) \quad \text { where } \quad q=p^{m} .
$$

For $p>3$, the Green functions have been determined by Beynon-Spaltenstein [1]. For $p=2,3$, the Green functions are explicitly computed by Malle [28] and Porsch [33. Since the results for $p=3$ have never been published, and since we also need them when dealing with the twisted case, we will provide here an independent verification of Porsch's results.

6.1. Critical unipotent classes for $p=3$. Assume from now on that $p=3$. We have $|\operatorname{Irr}(W)|=25$ and the character table of $W$ is available in CHEVIE. Now $F$ acts trivially on $W$ and $\gamma: W \rightarrow W$ is the identity. Consequently, $\operatorname{Irr}(W)=\operatorname{Irr}(W)^{\gamma}$. By Mizuno [31], there are 21 unipotent classes of $G$, which are all $F_{3}$-stable. Furthermore, for each unipotent class $C$, there exists an element $u_{0} \in C$ such that $F_{3}\left(u_{0}\right)=u_{0}$ and $F_{3}$ acts trivially on $A\left(u_{0}\right)$; see [31, Prop. 6.1]. Thus, condition (\$) in Section 3 holds. The Springer correspondence is explicitly described by Spaltenstein [41, p. 331]. As in Example 2.7, we run the function ICCTable which yields the coefficients $p_{E^{\prime}, E}$. By inspection of the output, we see that there is just one case which is not covered by the arguments in Remark 3.4; see Table 5 where the last two columns specify $E, E_{0} \in \operatorname{Irr}(W)$ such that $\iota_{G}\left(E_{0}\right)=\left(C, \overline{\mathbb{Q}}_{\ell}\right)$ and $\iota_{G}(E)=(C, \mathscr{E})$ with $\mathscr{E} \neq \overline{\mathbb{Q}}_{\ell}$.

TABLE 5. The critical unipotent class for type $E_{6}$ with $p=3$

\begin{tabular}{cccccc}
\hline$C$ & $\operatorname{dim} C_{G}(u)$ & $A(u)$ & $\mid C_{G}(u)^{F}$ & $E_{0}$ & $E$ \\
\hline$E_{6}\left(a_{3}\right)$ & 12 & $\mathbb{Z} / 2 \mathbb{Z}$ & $2 q^{12}, 2 q^{12}$ & $30_{3}$ & $15_{5}$ \\
\hline
\end{tabular}

In the table, we use the notation of Spaltenstein [41] for $\operatorname{Irr}(W)$, which is just a slight variation of Carter [3, §13.2] (or CHEVIE); for example, the representation $30_{3}$ is denoted by $\phi_{30,3}$ in [3, p. 415].

6.2. The class $E_{6}\left(a_{3}\right)$. Let $C$ be the unipotent class denoted by $E_{6}\left(a_{3}\right)$. (Note that Mizuno uses the notation $A_{5}+A_{1}$ for this class.) Since $A(u) \cong \mathbb{Z} / 2 \mathbb{Z}$ for $u \in C$, we are in the situation of Example 3.5, with $E_{0}=30_{3}$ and $E=15_{5}$. The following argument is analogous to that in $\$ 5.2$. We already know that there exists some $u_{0} \in C^{F}$ such that $F$ acts trivially on $A\left(u_{0}\right)$ and $\delta_{30_{3}}=\delta_{15_{5}}$. Using the 
output of ICCTable and the argument in Remark 3.4(a), we have $\delta_{30_{3}}=1$ and, hence, also $\delta_{15_{5}}=1$. The only remaining problem is to identify $u_{0}$ in a given list of class representatives. By Mizuno [31, Lemma 4.3], there is a choice of signs such that

$$
x_{18}:=x_{\alpha_{5}}( \pm 1) x_{\alpha_{4}}( \pm 1) x_{\alpha_{3}}( \pm 1) x_{\alpha_{1}}( \pm 1) x_{\alpha_{6}}( \pm 1) x_{\alpha_{1}+\alpha_{2}+\alpha_{3}+\alpha_{4}+\alpha_{5}+\alpha_{6}}( \pm 1) \in C .
$$

But then we check again that all elements as above, for all possible choices of the signs, are conjugate under elements of $T_{0}^{F_{3}}$. Thus, we may assume that all signs are +1 . Then we consider the signs $\delta_{30_{3}}$ and $\delta_{15_{5}}$ with respect to $x_{18}$. Since $\iota_{G}\left(30_{3}\right)=\left(C, \overline{\mathbb{Q}}_{\ell}\right)$, we already know that $\delta_{30_{3}}=1$. Since $x_{18} \in C^{F_{3}}$, we can apply Remark 3.7. Thus, it will be sufficient to determine $\delta_{15_{5}}$ in the special case where $m=1$. Using the output of ICCTable, we find the coefficients $\tilde{p}_{E^{\prime}}$ in $\$ 2.8$. This yields the formula

$$
Q_{1}\left(x_{18}\right)=\left(30 q^{3}+20 q^{2}+6 q+1\right) Y_{30_{3}}\left(x_{18}\right)+\left(15 q^{3}+6 q^{2}\right) Y_{15_{5}}\left(x_{18}\right) .
$$

Setting $q=3$, we obtain $Q_{1}\left(x_{18}\right)=1009+459 \delta_{15_{5}} \in\{1468,550\}$. By an explicit computation counting coset representatives, we find that $Q_{1}\left(x_{18}\right)=1468$. (In the setting of Lemma 2.9, we just need to look at sets $Q_{1, w}\left(x_{18}\right)$ where $l(w) \leqslant 12$.) Thus, indeed, $u_{0}:=x_{18} \in C$ is a representative with respect to which we have $\delta_{30_{3}}=\delta_{15_{5}}=1$ (regardless of the choice of a Chevalley basis).

6.3. A different representative for $E_{6}\left(a_{3}\right)$. Let $C$ be as above, but now consider the element

$$
x_{18}^{\prime}:=x_{\alpha_{1}}(1) x_{\alpha_{6}}(1) x_{\alpha_{3}}(1) x_{\alpha_{5}}(1) x_{\alpha_{4}}(1) x_{\alpha_{1}+\alpha_{2}+\alpha_{3}+\alpha_{4}+\alpha_{5}+\alpha_{6}}(1) \in G^{F_{3}} .
$$

(This will be useful in the following section, when we consider twisted groups of type $E_{6}$.) We claim that $x_{18}, x_{18}^{\prime}$ are conjugate in $G^{F_{3}}$. First we check that, in the adjoint representation, $x_{18}^{\prime}$ has Jordan blocks of sizes $9^{4}, 7,6^{4}, 3^{3}, 2$. Hence, we have $x_{18}^{\prime} \in C$; see [14, Table 6]. Furthermore, we check that all the elements

$$
x_{\alpha_{1}}( \pm 1) x_{\alpha_{6}}( \pm 1) x_{\alpha_{3}}( \pm 1) x_{\alpha_{5}}( \pm 1) x_{\alpha_{4}}( \pm 1) x_{\alpha_{1}+\alpha_{2}+\alpha_{3}+\alpha_{4}+\alpha_{5}+\alpha_{6}}( \pm 1)
$$

are conjugate under elements of $T_{0}^{F^{3}}$. As above, we set $q=3$ and compute that $Q_{1}\left(x_{18}^{\prime}\right)=1468$. Thus, $x_{18}^{\prime}$ must be conjugate in $G^{F_{3}}$ to $x_{18}$ (regardless of the choice of a Chevalley basis). Furthermore, if we take $x_{18}^{\prime}$ as the chosen representative in $C^{F}$, then the correponding signs $\delta_{30_{3}}$ and $\delta_{15_{5}}$ will again be equal to 1 .

6.4. Improving efficiency. We have $|W|=51840$ and there are 8335 elements $w \in W$ such that $l(w) \leqslant 12$. So, a priori, in 6.2 we would have to look at

$$
\sum_{w \in W, l(w) \leqslant 12} 3^{l(w)}=1569060811
$$

coset representatives in order to obtain that $Q_{1}\left(x_{18}\right)=1468$. Since we only need to establish the estimate $Q_{1}\left(x_{18}\right)>550$, we can try to reduce the number of elements $w \in W$ to consider, as follows.

Let $v \in U_{w}^{F}$ for some $w \in W$. Writing $v$ as a product of terms $x_{\alpha}(t)$ where $\alpha \in \Phi^{+}$and $t \in k$, and using Chevalley's commutator relations, we see that

$$
\begin{aligned}
v^{-1} x_{18} v=x_{\alpha_{5}}( \pm 1) x_{\alpha_{4}}( \pm 1) x_{\alpha_{3}}( \pm 1) x_{\alpha_{1}}( \pm 1) x_{\alpha_{6}}( \pm 1) \\
\quad \times \text { product of terms } x_{\alpha}(t) \text { where } \alpha \in \Phi^{+} \text {and } \alpha \neq \alpha_{i} \text { for all } i .
\end{aligned}
$$


Now $v$ will only contribute to $\left|Q_{1, w}\left(x_{18}\right)\right|$ if $\dot{w}^{-1} v^{-1} x_{18} v \dot{w} \in B_{0}^{F}$. So we just consider those $w \in W$ such that $l(w) \leqslant 12$ and the roots $w\left(\alpha_{5}\right), w\left(\alpha_{4}\right), w\left(\alpha_{3}\right), w\left(\alpha_{1}\right), w\left(\alpha_{6}\right)$ are all positive. There are 47 such elements $w \in W$, accounting for only 4220491 cosets. It turns out that, already among these cosets, we find more than 550 ones that are fixed by $x_{18}$. (And a similar procedure works in all the other cases that we consider in Section 8 , where $|W|=2903040$ and such a reduction becomes even more important.)

Remark 6.5. By analogous arguments, we obtain an independent verification of the results of Malle [28] on the Green functions of $E_{6}\left(2^{m}\right)$.

\section{On the Green functions of twisted $E_{6}$ In CharaCteristic 3}

Throughout this section, let again $G$ be a simple algebraic group of (adjoint) type $E_{6}$. We have $G=\left\langle x_{\alpha}(t) \mid \alpha \in \Phi, t \in k\right\rangle$ where $\Phi$ is the root system of $G$ with respect to $T_{0}$. Let $\left\{\alpha_{i} \mid 1 \leqslant i \leqslant 6\right\}$ be the set of simple roots with respect to $B_{0}$, where the labelling is chosen as in Table 3. We assume that $G$ is defined and split over $\mathbb{F}_{p}$, with corresponding Frobenius map $F_{p}: G \rightarrow G$ such that $F_{p}(t)=t^{p}$ for all $t \in T_{0}$. Now we also consider the non-trivial graph automorphism $\tilde{\gamma}: G \rightarrow G$ of order 2 , such that $\tilde{\gamma}\left(B_{0}\right)=B_{0}$ and $\tilde{\gamma}\left(T_{0}\right)=T_{0}$. This induces the following permutation of the simple roots:

$$
\alpha_{1} \rightarrow \alpha_{6}, \quad \alpha_{2} \rightarrow \alpha_{2}, \quad \alpha_{3} \rightarrow \alpha_{5}, \quad \alpha_{4} \rightarrow \alpha_{4}, \quad \alpha_{5} \rightarrow \alpha_{3}, \quad \alpha_{6} \rightarrow \alpha_{1} .
$$

Let $m \geqslant 1$ and $q=p^{m}$. Then $G^{F}={ }^{2} E_{6}(q)$ where $F:=\tilde{\gamma} \circ F_{p}^{m}=F_{p}^{m} \circ \tilde{\gamma}$.

For $p>3$, the Green functions have been determined by Beynon-Spaltenstein [1. For $p=2$, the Green functions are explicitly computed by Malle [28]. To complete the picture, it remains to deal with the case $p=3$.

7.1. Root elements in $G^{F}$. Let $\mathfrak{g}$ be the Lie algebra of type $E_{6}$, realized as a subalgebra of $\operatorname{End}(M)$ as in Section 4, recall that $M$ comes equipped with a basis $\left\{u_{1}, \ldots, u_{6}\right\} \cup\left\{v_{\alpha} \mid \alpha \in \Phi\right\}$. Let $\Phi \rightarrow \Phi, \alpha \mapsto \alpha^{\dagger}$, be the permutation induced by the automorphism $\tilde{\gamma}: G \rightarrow G$. Then define a linear map $\tau: M \rightarrow M$ by

$$
u_{1} \mapsto u_{6}, \quad u_{2} \mapsto u_{2}, \quad u_{3} \mapsto u_{5}, \quad u_{4} \mapsto u_{4}, \quad u_{5} \mapsto u_{3}, \quad u_{6} \mapsto u_{1}, \quad \text { and } v_{\alpha} \mapsto v_{\alpha^{\dagger}}
$$

for all $\alpha \in \Phi$; note that $\tau^{2}=\mathrm{id}_{M}$. Then one simply checks that conjugation with $\tau$ inside $\operatorname{End}(M)$ defines the non-trivial graph automorphism of $\mathfrak{g}$. We shall assume throughout this section that $G=G_{k} \subseteq \mathrm{GL}(\bar{M})$ is realised as in 44.4 . Then $\tilde{\gamma}$ is also realised by conjugation with $\tau$ inside $\operatorname{GL}(\bar{M})$. Furthermore, consider the "canonical" Chevalley basis $\left\{\mathbf{e}_{\alpha}^{\epsilon} \mid \alpha \in \Phi\right\}$ of $\mathfrak{g}$. Then one also checks that $\tau \circ \mathbf{e}_{\alpha}^{\epsilon}=\mathbf{e}_{\alpha^{\dagger}}^{\epsilon} \circ \tau$ for all $\alpha \in \Phi$. In this situation, root elements for $G^{F}$ have a simple description as in [2, Prop. 13.6.3], that is, given $\alpha \in \Phi$ and $t \in k$, we have

$$
\begin{aligned}
x_{\alpha}(t) & \in G^{F} & & \text { if } \alpha^{\dagger}=\alpha \text { and } t^{q}=t, \\
x_{\alpha}(t) x_{\alpha^{\dagger}}\left(t^{q}\right) & \in G^{F} & & \text { if } \alpha^{\dagger} \neq \alpha \text { and } t^{q^{2}}=t .
\end{aligned}
$$

(Note that, in type $E_{6}$, we have $\alpha+\alpha^{\dagger} \notin \Phi$ for all $\alpha \in \Phi$; so we only have to consider cases (i) and (ii) of [2, Prop. 13.6.3].) It is then straightforward to adjust the program in $\$ 4.7$ to the present situation. 
7.2. Critical unipotent classes for $p=3$. Assume from now on that $p=3$. The induced automorphism $\gamma: W \rightarrow W$ is given by conjugation with the longest element $w_{0} \in W$. Consequently, $\operatorname{Irr}(W)=\operatorname{Irr}(W)^{\gamma}$. For each $E \in \operatorname{Irr}(W)$, we need to choose a map $\sigma_{E}: E \rightarrow E$ as in \$2.1. In CHEVIE, the "preferred" choice for $\sigma_{E}$ specified by Lusztig [19, 17.2] is taken. By [15, Lemma 20.16], all the 21 unipotent classes of $G$ are stable under $F_{3}$ and under $\tilde{\gamma}$. Further information about the classes is provided in [15, Table 22.2.3]. This shows that, for each unipotent class $C$, there exists some $u_{0} \in C^{F}$ such that $F$ acts trivially on $A\left(u_{0}\right)$. Thus, condition (\$) in Section 3 holds. As in Example 2.7, we run the function ICCTable which yields the coefficients $p_{E^{\prime}, E}$ :

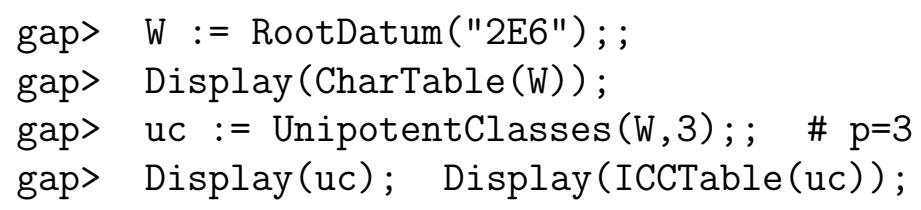

By inspection of the output, we see that there is only one case which is not covered by the arguments in Remark 3.4, exactly as in Section 6, Table 5.

7.3. The class $E_{6}\left(a_{3}\right)$ (twisted case). Let $C$ be the unipotent class denoted by $E_{6}\left(a_{3}\right)$. Since $A(u) \cong \mathbb{Z} / 2 \mathbb{Z}$ for $u \in C$, we are in the situation of Example 3.5, with $E_{0}=30_{3}$ and $E=15_{5}$. The following argument is analogous to that in $\$ 6.2$, but some additional care is needed because of the presence of the graph automorphism $\tilde{\gamma}$. We already know that there exists some $u_{0} \in C^{F}$ such that $F$ acts trivially on $A\left(u_{0}\right)$ and $\delta_{30_{3}}=\delta_{15_{5}}$. Using the output of ICCTable and the argument in Remark 3.4(a), we have $\delta_{30_{3}}=1$ and, hence, also $\delta_{15_{5}}=1$. Again, the only remaining problem is to identify $u_{0}$ in a given list of class representatives. We claim that, regardless of the choice of a Chevalley basis in the Lie algebra of $G$, we can take $u_{0}$ to be the element already considered in $\$ 6.3$.

$$
x_{18}^{\prime}:=x_{\alpha_{1}}(1) x_{\alpha_{6}}(1) x_{\alpha_{3}}(1) x_{\alpha_{5}}(1) x_{\alpha_{4}}(1) x_{\alpha_{1}+\alpha_{2}+\alpha_{3}+\alpha_{4}+\alpha_{5}+\alpha_{6}}(1) \in C .
$$

By 97.1 , we have $x_{18}^{\prime} \in G^{F}$; in fact, $x_{18}^{\prime}$ is fixed by both $F_{3}$ and $\tilde{\gamma}$. Conjugating by elements in $T_{0}^{F}$, one sees again that the $G^{F}$-conjugacy class of $x_{18}^{\prime}$ is well-defined, regardless of the choice of a Chevalley basis. We consider the signs $\delta_{30_{3}}$ and $\delta_{15_{5}}$ with respect to $x_{18}^{\prime}$. Since $\iota_{G}\left(30_{3}\right)=\left(C, \overline{\mathbb{Q}}_{\ell}\right)$, we already know that $\delta_{30_{3}}=1$. So it remains to show that $\delta_{15_{5}}=1$. Since $x_{18}^{\prime}$ is fixed by $F_{3}$ and by $\tilde{\gamma}$, we can apply the argument in [12, Remark 3.8]. This shows that

$$
\delta_{15_{5}} \delta_{15_{5}}^{\circ} \text { does not depend on } m \text {, }
$$

where $\delta_{15_{5}}^{\circ}$ is the sign from the untwisted case in Section 6. By $\$ \underline{6.3}$, we have $\delta_{15_{5}}^{\circ}=1$ for all $m$. Hence, we conclude that $\delta_{15_{5}}$ does not depend on $m$ either. So it will be sufficient to determine $\delta_{15_{5}}$ in the special case where $m=1$. Using the output of ICCTable, we find the coefficients $\tilde{p}_{E^{\prime}}$ in 92.8 . This yields the formula

$$
Q_{1}\left(x_{18}^{\prime}\right)=\left(10 q^{3}+4 q^{2}+2 q+1\right) Y_{30_{3}}\left(x_{18}^{\prime}\right)+\left(q^{3}-2 q^{2}\right) Y_{15_{5}}\left(x_{18}^{\prime}\right) \text {. }
$$

Setting $q=3$, we obtain $Q_{1}\left(x_{18}^{\prime}\right)=313+9 \delta_{15_{5}} \in\{322,304\}$. By an explicit computation counting coset representatives, we find $Q_{1}\left(x_{18}^{\prime}\right)=322$. (In the setting of Lemma 2.9, we just need to look at sets $Q_{1, w}\left(x_{18}^{\prime}\right)$ where $l(w) \leqslant 12$.) Thus, indeed, $x_{18}^{\prime} \in C$ is a representative with respect to which we have $\delta_{30_{3}}=\delta_{15_{5}}=1$.

Remark 7.3. By analogous arguments, we obtain an independent verification of the results of Malle [28] on the Green functions of ${ }^{2} E_{6}\left(2^{m}\right)$. 


\section{On the Green functions of type $E_{7}$ In Characteristics 2,3}

Throughout this section, let $G$ be a simple algebraic group of (adjoint) type $E_{7}$. We have $G=\left\langle x_{\alpha}(t) \mid \alpha \in \Phi, t \in k\right\rangle$ where $\Phi$ is the root system of $G$ with respect to $T_{0}$. Let $\left\{\alpha_{i} \mid 1 \leqslant i \leqslant 7\right\}$ be the set of simple roots with respect to $B_{0}$, where the labelling is chosen as in Table 3. We assume that $G$ is defined and split over $\mathbb{F}_{p}$, with corresponding Frobenius map $F_{p}: G \rightarrow G$ such that $F_{p}(t)=t^{p}$ for all $t \in T_{0}$. Let $F=F_{p}^{m}$ where $m \geqslant 1$. Then

$$
G^{F}=E_{7}(q) \quad \text { where } \quad q=p^{m} .
$$

For $p>3$, the Green functions have been determined by Beynon-Spaltenstein [1. To complete the picture, it remains to deal with the cases $p=2,3$. In the following, if $\alpha=\sum_{i=1}^{7} n_{i} \alpha_{i} \in \Phi$, we just write $x_{n_{1} n_{2} \ldots n_{7}}(t)$ instead of $x_{\alpha}(t)$.

8.1. Critical unipotent classes for $p=2,3$. Assume from now on that $p=2$ or $p=3$. We have $|\operatorname{Irr}(W)|=60$ and the character table of $W$ is available in CHEVIE. Now $F$ acts trivially on $W$ and $\gamma: W \rightarrow W$ is the identity. Consequently, $\operatorname{Irr}(W)=\operatorname{Irr}(W)^{\gamma}$. The unipotent classes of $G$ have been classified by Mizuno 32. Each unipotent class $C$ is $F$-stable and there exists an element $u_{0} \in C$ such that $F_{p}\left(u_{0}\right)=u_{0}$ and $F_{p}$ acts trivially on $A\left(u_{0}\right)$; see [32, Table 2]. Thus, condition (\$) in Section 3 holds. The Springer correspondence is explicitly described by Spaltenstein [41, p. 331-333]. As in Example 2.7, we run the function ICCTable which yields the coefficients $p_{E^{\prime}, E}$. By inspection of the output, we see that $p_{E^{\prime}, E_{1}} \in$ $\{0,1\}$ for all $E^{\prime} \in \operatorname{Irr}(W)$, where $E_{1}$ is the trivial representation of $W$. Hence, by Remark 3.4(a), we already have that

$$
\delta_{E_{0}}=1 \quad \text { for all } E_{0} \in \operatorname{Irr}(W) \text { such that } \iota_{G}\left(E_{0}\right)=\left(C, \overline{\mathbb{Q}}_{\ell}\right) .
$$

There are further cases which are not covered by the arguments in Remark 3.4(b); these are specified in Table 6 where, as before, the last two columns specify $E, E_{0} \in$ $\operatorname{Irr}(W)$ such that $\iota_{G}\left(E_{0}\right)=\left(C, \overline{\mathbb{Q}}_{\ell}\right)$ and $\iota_{G}(E)=(C, \mathscr{E})$ with $\mathscr{E} \approx \overline{\mathbb{Q}}_{\ell}$.

TABLE 6. Critical unipotent classes for type $E_{7}$ with $p=2,3$

\begin{tabular}{ccccccc}
\hline$p$ & $C$ & $\operatorname{dim} C_{G}(u)$ & $A(u)$ & $\left|C_{G}(u)^{F}\right|$ & $E_{0}$ & $E: \operatorname{dim} \mathscr{E}_{u}$ \\
\hline 2,3 & $E_{7}\left(a_{3}\right)$ & 13 & $\mathbb{Z} / 2 \mathbb{Z}$ & $2 q^{13}, 2 q^{13}$ & $56_{3}$ & $21_{6}$ \\
\hline 3 & $E_{7}\left(a_{4}\right)$ & 17 & $\mathbb{Z} / 2 \mathbb{Z}$ & $2 q^{17}, 2 q^{17}$ & $189_{5}$ & $15_{7}$ \\
\hline 2,3 & $E_{7}\left(a_{5}\right)$ & 21 & $\mathfrak{S}_{3}$ & $6 q^{21}, 2 q^{21}, 3 q^{21}$ & $315_{7}$ & $\begin{array}{c}280_{9}: 2 \\
35_{13}: 1\end{array}$ \\
\hline 2,3 & $E_{6}\left(a_{3}\right)$ & 23 & $\mathbb{Z} / 2 \mathbb{Z}$ & $2 q^{21}\left(q^{2}-1\right), 2 q^{21}\left(q^{2}-1\right)$ & $405_{8}$ & $189_{10}$ \\
\hline
\end{tabular}

In the table, we use the notation of Spaltenstein [41] for $\operatorname{Irr}(W)$, which is just a slight variation of Carter [3, §13.2] (or CHEVIE); for example, the representation $56_{3}$ is denoted by $\phi_{56,3}$ in [3, p. 416].

8.2. The class $E_{7}\left(a_{3}\right)$ for $p=2,3$. Let $C$ be the unipotent class denoted by $E_{7}\left(a_{3}\right)$. (Note that Mizuno uses the notation $D_{6}+A_{1}$ for this class.) Since $A(u) \cong$ $\mathbb{Z} / 2 \mathbb{Z}$ for $u \in C$, we are in the situation of Example 3.5, with $E_{0}=56_{3}$ and $E=21_{6}$. The following argument is analogous to that in 95.5 . We already know that there exists some $u_{0} \in C^{F}$ such that $F$ acts trivially on $A\left(u_{0}\right)$ and $\delta_{56_{3}}=\delta_{21_{6}}=1$. The only remaining problem is to identify $u_{0}$ in a given list of class representatives. 
Using Mizuno [31, Table 2], checking sizes of Jordan blocks, and arguing as in $\$ 5.5$, we may take

$$
\begin{aligned}
\tilde{u}^{ \pm}:= & x_{1000000}(1) x_{0101000}(1) x_{0011000}(1) x_{0101100}(1) \\
& \cdot x_{0111100}( \pm 1) x_{0000110}(1) x_{0000001}(1) \in C,
\end{aligned}
$$

regardless of the sign or the choice of a Chevalley basis. We consider the signs $\delta_{56_{3}}^{ \pm}$and $\delta_{21_{6}}^{ \pm}$with respect to $\tilde{u}^{ \pm}$. Since $\iota_{G}\left(56_{3}\right)=\left(C, \overline{\mathbb{Q}}_{\ell}\right)$, we already know that $\delta_{56_{3}}^{ \pm}=1$. Since $\tilde{u}^{ \pm} \in C^{F_{p}}$, we can apply Remark 3.7. Thus, it will be sufficient to determine $\delta_{21_{6}}^{ \pm}$in the special case where $m=1$. Using the output of ICCTable, and setting $q=2$ or $q=3$, we obtain the formulae

$$
\begin{aligned}
Q_{1}\left(\tilde{u}^{ \pm}\right) & =\left(56 q^{3}+27 q^{2}+7 q+1\right) Y_{56_{3}}\left(\tilde{u}^{ \pm}\right)+\left(21 q^{3}+7 q^{2}\right) Y_{21_{6}}\left(\tilde{u}^{ \pm}\right) \\
& =\left\{\begin{array}{cl}
571+196 \delta_{21_{6}} \in\{767,375\} & (q=2), \\
1777+630 \delta_{21_{6}} \in\{2407,1147\} & (q=3) .
\end{array}\right.
\end{aligned}
$$

(Of course, in general, the polyonomial expressions for the values of $Q_{1}$ will depend on whether $p=2$ or $p=3$, but for the classes in Table 6, they do coincide.) By an explicit computation counting coset representatives (see Lemma 2.9), we find that $Q_{1}\left(\tilde{u}^{ \pm}\right)$equals 767 if $p=2$, and 2407 if $p=3$. (If $p=2$, then we just need to look at sets $Q_{1, w}\left(\tilde{u}^{ \pm}\right)$where $l(w) \leqslant 15$ in order to find 767 cosets that are fixed; if $p=3$, then we just need to go up to $l(w) \leqslant 6$ in order to find strictly more than 1147 cosets that are fixed.) In particular, $\tilde{u}^{+}, \tilde{u}^{-}$are conjugate in $G^{F}$. Thus, indeed, $\tilde{u}^{+} \in C$ is a representative with respect to which we have $\delta_{56_{3}}=\delta_{21_{6}}=1$ (regardless of the choice of a Chevalley basis).

8.3. The class $E_{7}\left(a_{4}\right)$ for $p=3$. Let $p=3$ and $C$ be the unipotent class denoted by $E_{7}\left(a_{4}\right)$. (Note that Mizuno uses the notation $D_{6}\left(a_{1}\right)+A_{1}$ for this class.) Since $A(u) \cong \mathbb{Z} / 2 \mathbb{Z}$ for $u \in C$, we are in the situation of Example 3.5, with $E_{0}=189_{5}$ and $E=15_{7}$. The following argument is analogous to that in $\$ 5.5$. We already know that there exists some $u_{0} \in C^{F}$ such that $F$ acts trivially on $A\left(u_{0}\right)$ and $\delta_{189_{7}}=\delta_{15_{7}}=1$. The only remaining problem is to identify $u_{0}$ in a given list of class representatives. Using Mizuno [31, Table 2], checking sizes of Jordan blocks, and arguing as in 95.5 , we may take

$$
\begin{aligned}
\tilde{u}^{ \pm}:= & x_{1000000}(1) x_{0111000}(1) x_{0011100}(1) x_{0101100}(1) \\
& \cdot x_{0001110}(1) x_{0011110}( \pm 1) x_{0000011}(1) \in C,
\end{aligned}
$$

regardless of the sign or the choice of a Chevalley basis. We consider the signs $\delta_{189_{5}}^{ \pm}$ and $\delta_{15_{7}}^{ \pm}$with respect to $\tilde{u}^{ \pm}$. We already know that $\delta_{189_{5}}^{ \pm}=1$. Since $\tilde{u}^{ \pm} \in C^{F_{3}}$, we can apply Remark 3.7. Thus, it will be sufficient to determine $\delta_{15_{7}}$ in the special case where $m=1$. Using the output of ICCTable, we find the following formula.

$$
Q_{1}\left(\tilde{u}^{ \pm}\right)=\left(189 q^{5}+155 q^{4}+77 q^{3}+27 q^{2}+7 q+1\right) Y_{189_{5}}\left(\tilde{u}^{ \pm}\right)+15 q^{5} Y_{15_{7}}\left(\tilde{u}^{ \pm}\right) .
$$

Setting $q=3$, we obtain that $Q_{1}\left(\tilde{u}^{ \pm}\right)=60826+3645 \delta_{15_{7}} \in\{64471,57181\}$. By an explicit computation counting coset representatives, we find that $Q_{1}\left(\tilde{u}^{ \pm}\right)=64471$. (We just need to look at sets $Q_{1, w}\left(\tilde{u}^{ \pm}\right)$where $l(w) \leqslant 16$ in order to find strictly more than 57181 cosets that are fixed by $\tilde{u}^{ \pm}$.) Thus, $\tilde{u}^{+}$and $\tilde{u}^{-}$are conjugate in $G^{F}$ and, indeed, $\tilde{u}^{+} \in C$ is a representative with respect to which we have $\delta_{15_{7}}=1$ (regardless of the choice of a Chevalley basis). 
8.4. The class $E_{7}\left(a_{5}\right)$ for $p=2,3$. Let $C$ be the unipotent class denoted by $E_{7}\left(a_{5}\right)$. (Note that Mizuno uses the notation $D_{6}\left(a_{2}\right)+A_{1}$ for this class.) We have $A(u) \cong \mathfrak{S}_{3}$ for $u \in C$. The set $C^{F}$ splits into three classes in $G^{F}$, with centraliser orders $6 q^{21}, 2 q^{21}, 3 q^{21}$. Thus, up to conjugation by elements in $G^{F}$, there is a unique $u_{0} \in C^{F}$ such that $\left|C_{G}\left(u_{0}\right)^{F}\right|=6 q^{21}$ and $F$ acts trivially on $A\left(u_{0}\right)$. Now, via the Springer correspondence, there are three irreducible representations of $W$ associated with $C$. These are $315_{7}, 280_{9}, 35_{13}$; see Table 6. We already know that $\delta_{315_{7}}=1$. Now $u_{0}$ can be chosen to be fixed by $F_{p}$; see the explicit expression in [32, Table 2]. So, by Remark [3.7, it is sufficient to determine $\delta_{280_{9}}, \delta_{35_{13}}$ in the special case where $m=1$. The following argument is analogous to that in $\$ 5.4$,

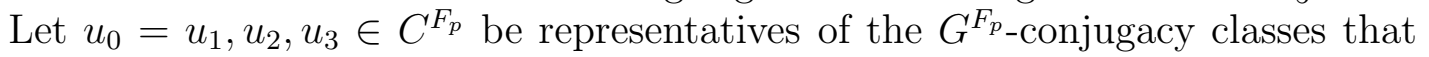
are contained in $C^{F_{p}}$, and let $a_{1}, a_{2}, a_{3} \in A\left(u_{0}\right)$ be corresponding representatives of the conjugacy classes of $A\left(u_{0}\right)$, where the notation is such that $a_{2}$ corresponds to a transposition in $\mathfrak{S}_{3} \cong A\left(u_{0}\right)$ and $a_{3}$ to a 3 -cycle. Using the output of ICCTable, and setting $q=2$ or $q=3$, we find the formula

$$
\begin{aligned}
& Q_{1}\left(u_{i}\right)=\left(315 q^{7}+483 q^{6}+350 q^{5}+182 q^{4}+77 q^{3}+27 q^{2}+7 q+1\right) Y_{315_{7}}\left(u_{i}\right) \\
& +\left(280 q^{7}+330 q^{6}+161 q^{5}+48 q^{4}+7 q^{3}\right) Y_{280_{9}}\left(u_{i}\right)+\left(35 q^{7}+21 q^{6}\right) Y_{35_{13}}\left(u_{i}\right) \\
& =\left\{\begin{array}{cc}
86083 Y_{315_{7}}\left(u_{i}\right)+62936 Y_{280_{9}}\left(u_{i}\right)+5824 Y_{35_{13}}\left(u_{i}\right) & (q=2), \\
1143148 Y_{315_{7}}\left(u_{i}\right)+896130 Y_{280_{9}}\left(u_{i}\right)+91854 Y_{35_{13}}\left(u_{i}\right) & (q=3),
\end{array}\right.
\end{aligned}
$$

for $1 \leqslant i \leqslant 3$. Now, up to the signs $\delta_{280_{9}}$ and $\delta_{35_{13}}$, the values of the $Y$-functions on $u_{i}$ are given by character values of $\mathfrak{S}_{3}$; see Remark 3.2 . Thus, we obtain

$$
Q_{1}\left(u_{1}\right)=\left\{\begin{array}{cc}
86083+2 \cdot 62936 \delta_{280_{9}}+5824 \delta_{35_{13}} & (q=2) \\
1143148+2 \cdot 896130 \delta_{280_{9}}+91854 \delta_{35_{13}} & (q=3) .
\end{array}\right.
$$

Since $Q_{1}\left(u_{1}\right) \geqslant 0$, this already forces that $\delta_{280_{9}}=1$ in both cases. We claim that we also have $\delta_{3513}=1$. For this purpose, we consider the values at $u_{3}$ :

$$
Q_{1}\left(u_{3}\right)=\left\{\begin{array}{cc}
86083-62936+5824 \delta_{35_{13}}=23147+5824 \delta_{35_{13}} & (q=2), \\
1143148-896130+91854 \delta_{35_{13}}=247018+91854 \delta_{35_{13}} & (q=3) .
\end{array}\right.
$$

By Mizuno [32, Lemma 21], there is a choice of signs $\varepsilon_{i}= \pm 1$ such that

$$
\begin{aligned}
y_{46}:= & x_{1011000}\left(\varepsilon_{1}\right) x_{0111000}\left(\varepsilon_{2}\right) x_{0011100}\left(\varepsilon_{3}\right) x_{0101100}\left(\varepsilon_{4}\right) \\
& \cdot x_{0001110}\left(\varepsilon_{5}\right) x_{0000111}\left(\varepsilon_{6}\right) x_{1111100}\left(\varepsilon_{7}\right) x_{1111110}\left(\varepsilon_{8}\right) \in C
\end{aligned}
$$

and $\left|C_{G}\left(y_{46}\right)^{F}\right|=3 q^{21}$. Then $y_{46}$ will be conjugate to $u_{3}$ in $G^{F}$.

If $q=2$, then $\varepsilon_{i}=1$ for all $i$, and the above formula shows that $Q_{1}\left(u_{3}\right) \in$ $\{28971,17323\}$. By an explicit computation counting coset representatives (see Lemma 2.9) , we find that $Q_{1}\left(y_{46}\right)=17323$. (We just need to look at sets $Q_{1, w}\left(y_{46}\right)$ where $l(w) \leqslant 13$.) Hence, $\delta_{35_{13}}=1$, as claimed.

Now assume that $p=3$. Then $Q_{1}\left(u_{3}\right) \in\{338872,155164\}$. Now we simply consider all elements $y_{46}$ as above, for all posssible choices of the signs $\varepsilon_{i}$. For each such choice, $y_{46}$ has Jordan blocks of sizes $9^{9}, 7,6^{2}, 53,3^{6}$ and, hence, $y_{46} \in C$; see [14, Table 8]. Furthermore, we find that $Q_{1}\left(y_{46}\right)=338872$ in each case. (We just need to look at sets $Q_{1, w}\left(y_{46}\right)$ where $l(w) \leqslant 8$.) Hence, $\delta_{35_{13}}=1$, as claimed.

8.5. The class $E_{6}\left(a_{3}\right)$ for $p=2,3$. Let $C$ be the unipotent class denoted by $E_{6}\left(a_{3}\right)$. (Note that Mizuno uses the notation $\left(A_{5}+A_{1}\right)^{\prime}$ for this class.) Since $A(u) \cong \mathbb{Z} / 2 \mathbb{Z}$ for $u \in C$, we are in the situation of Example 3.5, with $E_{0}=405_{8}$ 
and $E=189_{10}$. The following argument is analogous to that in $\$ 5.2$. We already know that there exists some $u_{0} \in C^{F}$ such that $F$ acts trivially on $A\left(u_{0}\right)$ and $\delta_{405_{8}}=\delta_{189_{10}}=1$. The only remaining problem is to identify $u_{0}$ in a given list of class representatives. Using Mizuno [31, Table 2], checking sizes of Jordan blocks, and arguing as in $\$ 5.2$, we may take

$$
\tilde{u}:=x_{1010000}(1) x_{0111000}(1) x_{0011100}(1) x_{1111000}(1) x_{0101110}(1) x_{0001111}(1) \in C,
$$

regardless of the choice of a Chevalley basis. We consider the signs $\delta_{405_{8}}$ and $\delta_{189_{10}}$ with respect to $\tilde{u}$. We already know that $\delta_{405_{8}}=1$. Since $\tilde{u} \in C^{F_{3}}$, we can apply Remark 3.7. Thus, it will be sufficient to determine $\delta_{189_{10}}$ in the special case where $m=1$. Using the output of ICCTable, we find the following formula.

$$
\begin{aligned}
& Q_{1}(\tilde{u})=\left(405 q^{8}+973 q^{7}+933 q^{6}+532 q^{5}+230 q^{4}+84 q^{3}+27 q^{2}+7 q+1\right) Y_{405_{8}}(\tilde{u}) \\
& +\left(189 q^{8}+420 q^{7}+351 q^{6}+161 q^{5}+48 q^{4}+7 q^{3}\right) Y_{189_{10}}(\tilde{u}) \\
& =\left\{\begin{aligned}
309435+130584 \delta_{189_{10}} & \in\{440019,178851\} & & (q=2), \\
5615752+2457648 \delta_{189_{10}} & \in\{8073400,3158104\} & & (q=3) .
\end{aligned}\right.
\end{aligned}
$$

On the other hand, using Lemma [2.9, we can try to directly compute $Q_{1}(\tilde{u})$.

If $p=2$, then we just need to look at sets $Q_{1, w}(\tilde{u})$ where $l(w) \leqslant 12$ in order to find strictly more than 178851 cosets that are fixed. A comparison with the above formula shows that we must have $\delta_{189_{10}}=1$. If $p=3$, then we need to go up to $l(w) \leqslant 13$ in order to find strictly more than 3158104 cosets that are fixed. (This is the hardest case for type $E_{7}$; the computation requires less than $4 \mathrm{~GB}$ of main memory but takes about a week on a standard computer, even with efficiency improvements as in 6.4 .) So we also have $\delta_{189_{10}}=1$ in this case.

\section{On the Green functions of type $E_{8}$ in Characteristics 2}

In this final section, let $G$ be a simple algebraic group of type $E_{8}$ and $F_{p}: G \rightarrow G$ be a split Froebnius map as before. Let $F=F_{p}^{m}$ where $m \geqslant 1$. Then

$$
G^{F}=E_{8}(q) \quad \text { where } \quad q=p^{m} .
$$

For $p>5$, the Green functions have been determined by Beynon-Spaltenstein [1]. Here, we will not be able to complete the computation of the Green functions for the cases where $p=2,3,5$. But we can at least show that the very particular case mentioned in Remark 3.4 also occurs for $p=2$. So assume from now on that $p=2$.

We have $|\operatorname{Irr}(W)|=112$ and the character table of $W$ is available in CHEVIE. Now $F$ acts trivially on $W$ and $\gamma: W \rightarrow W$ is the identity. Consequently, $\operatorname{Irr}(W)=$ $\operatorname{Irr}(W)^{\gamma}$. The unipotent classes of $G$ have been classified by Mizuno [32]. The Springer correspondence is explicitly described by Spaltenstein [41, p. 333-336]. The "very particular" case is related to the unipotent class $C$ specified as follows.

\begin{tabular}{cccccc}
\hline$C$ & $\operatorname{dim} C_{G}(u)$ & $A(u)$ & $\left|C_{G}(u)^{F}\right|$ & $E_{0}$ & $E: \operatorname{dim} \mathscr{E}_{u}$ \\
\hline$E_{8}\left(b_{6}\right)$ & 28 & \multirow{2}{*}{28} & \multirow{2}{*}{$6 q^{28}, 2 q^{28}, 3 q^{28}$} & $2240_{10}$ & $\begin{array}{l}175_{12}: 2 \\
840_{13}: 1\end{array}$ \\
\hline
\end{tabular}

where, as before, the last two columns specify $E, E_{0} \in \operatorname{Irr}(W)$ such that $\iota_{G}\left(E_{0}\right)=$ $\left(C, \overline{\mathbb{Q}}_{\ell}\right)$ and $\iota_{G}(E)=(C, \mathscr{E})$ with $\mathscr{E} \not \overline{\mathbb{Q}}_{\ell}$. (Note that Mizuno uses the notation $D_{8}\left(a_{3}\right)$ for this class; furthermore, the same conventions for the notation of $\operatorname{Irr}(W)$ apply as in Section 8.) Up to conjugation by elements in $G^{F}$, there is a unique 
$u_{0} \in C^{F}$ such that $\left|C_{G}\left(u_{0}\right)^{F}\right|=6 q^{28}$ and $F$ acts trivially on $A\left(u_{0}\right)$. By Mizuno [32, Lemma 53], such a representative is given by

$$
\begin{aligned}
& u_{0}:=z_{77}=x_{11110000}(1) x_{10111000}(1) x_{01111000}(1) x_{00111100}(1) \\
& \cdot x_{01011100}(1) x_{00011110}(1) x_{00000011}(1) x_{00000111}(1) .
\end{aligned}
$$

(As before, if $\alpha=\sum_{i=1}^{8} n_{i} \alpha_{i} \in \Phi$, we just write $x_{n_{1} n_{2} \ldots n_{8}}(t)$ instead of $x_{\alpha}(t)$.) We consider the corresponding signs $\delta_{E}$ with respect to $u_{0}$; we claim that

$$
\delta_{2240_{10}}=1 \quad \text { and } \quad \delta_{840_{13}}=(-1)^{m} \quad\left(q=2^{m}\right) .
$$

This is seen as follows. As before, since $u_{0} \in C^{F_{2}}$, it is sufficient to determine the signs in the special case where $m=1$ (see Remark 3.7). As in Example 2.7, we run the function ICCTable which yields the coefficients $p_{E^{\prime}, E}$. By inspection of the output, and using the argument in Remak 3.4(a), we already see that $\delta_{2240_{10}}=1$.

We now follow the argument in $\$ 8.4$. Let $u_{0}=u_{1}, u_{2}, u_{3} \in C^{F_{2}}$ be representatives of the $G^{F_{2}}$-conjugacy classes that are contained in $C^{F_{2}}$, and let $a_{1}, a_{2}, a_{3} \in A\left(u_{0}\right)$ be corresponding representatives of the conjugacy classes of $A\left(u_{0}\right)$, where the notation is such that $a_{2}$ corresponds to a transposition in $\mathfrak{S}_{3} \cong A\left(u_{0}\right)$ and $a_{3}$ to a 3 -cycle. By Mizuno [32, Lemma 53], such representatives are given by

$$
\begin{aligned}
& u_{2}:=z_{78}=z_{77} x_{00001111}(1), \\
& u_{3}:=z_{79}=x_{11110000}(1) x_{10111000}(1) x_{01111000}(1) x_{00111100}(1) x_{01011100}(1) \\
& \cdot x_{00011110}(1) x_{00000001}(1) x_{00000111}(1) x_{00001111}(1) .
\end{aligned}
$$

Using the output of ICCTable, and setting $q=2$, we find the formula

$$
\begin{aligned}
Q_{1}\left(u_{i}\right)=\left(2240 q^{10}+3688 q^{9}+3444 q^{8}+2360 q^{7}+1351 q^{6}\right. & \\
& \left.+672 q^{5}+294 q^{4}+112 q^{3}+35 q^{2}+8 q+1\right) Y_{2240_{10}}\left(u_{i}\right) \\
& +175 q^{10} Y_{175_{12}}\left(u_{i}\right)+\left(840 q^{10}+650 q^{9}+160 q^{8}\right) Y_{840_{13}}\left(u_{i}\right) \\
= & 5479485 Y_{2240_{10}}\left(u_{i}\right)+179200 Y_{175_{12}}\left(u_{i}\right)+1233920 Y_{840_{13}}\left(u_{i}\right) \quad(q=2) .
\end{aligned}
$$

for $1 \leqslant i \leqslant 3$. Up to the signs $\delta_{175_{12}}$ and $\delta_{840_{13}}$, the values of the $Y$-functions on $u_{i}$ are given by character values of $\mathfrak{S}_{3}$; see Remark 3.2. Thus, for $q=2$, we obtain

$$
\begin{aligned}
& Q_{1}\left(u_{1}\right)=5479485+2 \cdot 179200 \delta_{175_{12}}+1233920 \delta_{840_{13}}, \\
& Q_{1}\left(u_{2}\right)=5479485-1233920 \delta_{840_{13}}, \\
& Q_{1}\left(u_{3}\right)=5479485-179200 \delta_{175_{12}}+1233920 \delta_{840_{13}} .
\end{aligned}
$$

Assume, if possible, that $\delta_{840_{13}}=1$. Then we would have $Q_{1}\left(u_{2}\right)=5479485-$ $1233920=4245565$ for $q=2$. On the other hand, running through all sets $Q_{1, w}\left(z_{78}\right)$ (as in Lemma 2.9) where $l(w) \leqslant 23$, we already find 4047101 cosets that are fixed by $z_{78}$. Running also through sets $Q_{1, w}\left(z_{78}\right)$ where $l(w)=24$, we find further 305856 cosets that are fixed. Thus, we have $Q_{1}\left(u_{2}\right)>4245565$ and so we conclude that $\delta_{840_{13}}=-1$, as claimed.

The total running time for these computations is about 1 year, even with efficiency improvements as in $\$ \underline{6.4}$. (It already takes 4 months just to deal with those sets $Q_{1, w}\left(z_{78}\right)$ where $l(w)=24$.) Everything is much slower than in the previous cases because, for type $E_{8}$, we only have at our disposal the 248-dimensional adjoint representation, whereas for type $E_{7}$ we could use the 56-dimensional minuscule weight representation. However, distributing the task over a small number of 
(independent) standard desktop computers with altogether 30 processors, we could manage to complete the computations in 2 weeks. - Once $\delta_{840_{13}}$ is determined, we obtain $Q_{1}\left(u_{1}\right)=5479495-1233920+2 \cdot 179200 \delta_{175_{12}} \in\{4603965,3887165\}$ (for $q=2$ ). If we could find strictly more than 3887165 cosets that are fixed by $z_{77}$, then we would be able to conclude that $\delta_{175_{12}}=1$. However, this appears to be even more difficult computationally, the reason being that the difference between the lower and the upper bound for the value of $Q_{1}$ is much smaller than in the previous case. (So we would need to find almost all cosets that are fixed by $z_{77}$.)

All this clearly indicates that type $E_{8}$ is not completely out of reach, but some more sophisticated algorithms are certainly required in order to deal with the remaining open cases (especially for $p=3,5$ ). An independent verification of the above results would also be highly desirable.

\section{REFERENCES}

[1] W. M. Beynon and N. Spaltenstein, Green functions of finite Chevalley groups of type $E_{n}$ $(n=6,7,8)$, J. Algebra 88 (1984), 584-614.

[2] R. W. Carter, Simple groups of Lie type, Wiley, New York, 1972; reprinted 1989 as Wiley Classics Library Edition.

[3] R. W. Carter, Finite groups of Lie type: Conjugacy classes and complex characters, Wiley, New York, 1985.

[4] A. M. Cohen, S. H. Murray and D. E. Taylor, Computing in groups of Lie type, Math. Comp. 73 (2004), 1477-1498.

[5] P. Deligne and G. Lusztig, Representations of reductive groups over finite fields, Annals Math. 103 (1976), 103-161.

[6] The GAP Group, GAP - Groups, Algorithms, and Programming, Version 4.10.0, 2018, (https://www.gap-system.org).

[7] M. Geck, On the average values of the irreducible characters of finite groups of Lie type on geometric unipotent classes, Doc. Math. J. DMV 1 (1996), 293-317 (electronic).

[8] M. Geck, On the construction of semisimple Lie algebras and Chevalley groups, Proc. Amer. Math. Soc. 145 (2017), 3233-3247.

[9] M. Geck, Minuscule weights and Chevalley groups, in: Finite Simple Groups: Thirty Years of the Atlas and Beyond (Celebrating the Atlases and Honoring John Conway, November 2-5, 2015 at Princeton University), pp. 159-176, Contemporary Math., vol. 694, Amer. Math. Soc., 2017.

[10] M. Geck, ChevLie - Constructing Lie algebras and Chevalley groups in GAP, July 2016, available at http://www.math.rwth-aachen.de/ CHEVIE/contrib.html.

[11] M. Geck, On the values of unipotent characters in bad characteristic, Rend. Cont. Sem. Mat. Univ. Padova (2019), online first, DOI:10.4171/RSMUP/14.

[12] M. Geck, Green functions and Glauberman degree-divisibility; preprint (April, 2019); see arXiv:1904.04586.

[13] M. Geck, G. Hiss, F. Lübeck, G. Malle, and G. Pfeiffer, CHEVIE-A system for computing and processing generic character tables, Appl. Algebra Engrg. Comm. Comput. 7 (1996), 175-210; electronically available at http://www.math.rwth-aachen.de/ CHEVIE

[14] R. Lawther, Jordan block sizes of unipotent elements in exceptional algebraic groups, Comm. Algebra 23 (1995), 4125-4156.

[15] M. W. Liebeck and G. M. Seitz, Unipotent and nilpotent classes in simple algebraic groups and Lie algebras, Math. Surveys and Monographs, vol. 180, Amer. Math. Soc., Providence, RI, 2012.

[16] G. Lusztig, Representations of finite Chevalley groups, C.B.M.S. Regional Conference Series in Mathematics, vol. 39, Amer. Math. Soc., Providence, RI, 1977.

[17] G. Lusztig, Characters of reductive groups over a finite field, Ann. Math. Studies 107, Princeton U. Press, 1984. 
[18] G. Lusztig, Intersection cohomology complexes on a reductive group, Invent. Math. $\mathbf{7 5}$ (1984), 205-272.

[19] G. Lusztig, Character sheaves IV, Adv. Math. 59 (1986), 1-63.

[20] G. Lusztig, Character sheaves V, Adv. Math. 61 (1986), 103-155.

[21] G. Lusztig, Introduction to character sheaves, in: The Arcata Conference on Representations of Finite Groups (Arcata, Calif., 1986), Proc. Sympos. Pure Math., 47, Part 1, Amer. Math. Soc., Providence, RI, 1987, pp. 164-179.

[22] G. Lusztig, Green functions and character sheaves, Ann. Math. 131 (1990), 355-408.

[23] G. Lusztig, Character sheaves on disconnected groups, IV, Represent. Theory 8 (2004), $145-178$.

[24] G. Lusztig, On the cleanness of cuspidal character sheaves, Mosc. Math. J. 12 (2012), 621-631.

[25] G. Lusztig, The canonical basis of the quantum adjoint representation, J. Comb. Alg. 1 (2017), 45-57.

[26] G. Lusztig and N. Spaltenstein, On the generalized Springer correspondence for classical groups, in: Algebraic groups and related topics, Adv. Stud. Pure Math. 6, North Holland and Kinokuniya 1985, pp. 289-316.

[27] G. Malle, Die unipotenten Charaktere von ${ }^{2} F_{4}\left(q^{2}\right)$. Comm. Algebra 18 (1990), 2361-2381.

[28] G. Malle, Green functions for groups of type $F_{4}$ and $E_{6}$ in characteristic 2, Comm. Algebra 21 (1993), 747-798.

[29] R. M. Marcelo and K. Shinoda, Values of the unipotent characters of the Chevalley group of type $F_{4}$ at unipotent elements, Tokyo J. Math. 18 (1995), 303-340.

[30] J. Michel, The development version of the CHEVIE package of GAP3, J. Algebra 435 (2015), 308-336. Webpage at https://webusers.imj-prg.fr/ jean.michel/chevie/chevie.html.

[31] K. Mizuno, The conjugate classes of Chevalley groups of type $E_{6}$, J. Fac. Sci. Univ. Tokyo Sect. IA Math. 24 (1977), 525-563.

[32] K. Mizuno, The conjugate classes of unipotent elements of the Chevalley groups $E_{7}$ and $E_{8}$, Tokyo J. Math. 3 (1980), 391-461.

[33] U. Porsch, Die Greenfunktionen der endlichen Gruppen $E_{6}(q), q=3^{n}$, Diplomarbeit, Universität Heidelberg, 1993.

[34] T. Shoji, The conjugacy classes of Chevalley groups of type $\left(F_{4}\right)$ over finite fields of characteristic $p \neq 2$. J. Fac. Sci. Univ. Tokyo Sect. IA Math. 21 (1974), 1-17.

[35] T. Shoji, On the Green polynomials of a Chevalley group of type $F_{4}$, Comm. Algebra 10 (1982), 505-543.

[36] T. Shoji, Green functions of reductive groups over a finite field, in: The Arcata Conference on Representations of Finite Groups (Arcata, Calif., 1986), Proc. Sympos. Pure Math., 47, Part 1, Amer. Math. Soc., Providence, RI, 1987, pp. 289-302.

[37] T. Shoji, Character sheaves and almost characters of reductive groups, Adv. Math. 111 (1995), 244-313.

[38] T. Shoji, Character sheaves and almost characters of reductive groups, II, Adv. Math. 111 (1995), 314-354.

[39] T. Shoji, Generalized Green functions and unipotent classes for finite reductive groups, I, Nagoya Math. J. 184 (2006), 155-198.

[40] T. Shoji, Generalized Green functions and unipotent classes for finite reductive groups, II, Nagoya Math. J. 188 (2007), 133-170.

[41] N. Spaltenstein, On the generalized Springer correspondence for exceptional groups, in: Algebraic groups and related topics, Adv. Stud. Pure Math. 6, North Holland and Kinokuniya, 1985, pp. 317-338.

[42] R. Steinberg, Lectures on Chevalley groups. Mimeographed notes, Department of Math., Yale University, 1967. Now available as vol. 66 of the University Lecture Series, Amer. Math. Soc., Providence, R.I., 2016.

IAZ - Lehrstuhl für Algebra, Universität Stuttgart, Pfaffenwaldring 57, D70569 Stuttgart, Germany

E-mail address: meinolf.geck@mathematik.uni-stuttgart.de 